\title{
Scattering of thermal He beams by crossed atomic and molecular beams. II. The He-Ar van der Waals potentia|a) $^{\text {a }}$
}

\author{
Mark Keil, b) John T. Slankas, and Aron Kuppermann \\ Arthur Amos Noyes Laboratory of Chemical Physics, ${ }^{c}$ California Institute of Technology, Pasadena, \\ California 91125 \\ (Received 17 April 1978)

\begin{abstract}
Differential cross sections for He-Ar scattering at room temperature have been measured. The experimental consistency of these measurements with others performed in different laboratories is demonstrated. Despite this consistency, the present van der Waals well depth of $1.78 \mathrm{meV}$, accurate to $10 \%$, is smaller by $20 \%$ to $50 \%$ than the experimental values obtained previously. These discrepancies are caused by differences between the assumed mathematical forms or between the assumed dispersion coefficients of the potentials used in the present paper and those of previous studies. Independent investigations have shown that the previous assumptions are inappropriate for providing accurate potentials from fits to experimental differential cross section data for He-Ar. We use two forms free of this inadequacy in the present analysis: a modified version of the Simons-Parr-Finlan-Dunham (SPFD) potential, and a double Morse-van der Waals $\left(\mathrm{M}^{2} \mathrm{SV}\right.$ ) type of parameterization. The resulting He-Ar potentials are shown to be equal to within experimental error, throughout the range of interatomic distances to which the scattering data are sensitive. The SPFD or $\mathrm{M}^{2} \mathrm{SV}$ potentials are combined with a repulsive potential previously determined exclusively from fits to gas phase bulk properties. The resulting potentials, valid over the extended range of interatomic distances $r \leq 2.4 \AA$, are able to reproduce all these bulk properties quite well, without adversely affecting the quality of the fits to the DCS.
\end{abstract}

\section{INTRODUCTION}

Recent experimental and theoretical efforts directed towards the determination of interatomic potentials for rare gas van der Waals interactions have improved remarkably the characterization and understanding of such potentials. ${ }^{1}$ Early investigations of homonuclear interactions did much to elucidate the nature of their short- ${ }^{2,3}$ and long-range ${ }^{4}$ components, while later results for the same systems provided accurate information about the important attractive minimum region. ${ }^{5-7}$ As a result of these concerted efforts, the van der Waals potentials for the homonuclear rare gas pairs have been determined quite accurately; experimental data of many different kinds may be predicted satisfactorily from these potentials. ${ }^{1}$

The outstanding success with which these homonuclear potentials have been determined unfortunately is not attained for the more complicated case of heteronuclear interactions. ${ }^{1}$ This is of course partially due to less intensive investigations of the heteronuclear rare gas pairs as compared to those of the homonuclear ones; there are also significant experimental difficulties. For example, ${ }^{8,9}$ macroscopic heteronuclear interaction properties may be derived only indirectly from corresponding measurements of the mixtures, due to contributions of like-atom interactions. ${ }^{10}$ Historically, the incentive for the extensive development of various "combination rules", only some of which are soundly

\footnotetext{
a) This work was supported in part by a contract (EY-76-S-03767) from the Department of Energy. Report Code: CALT767P4-167.

${ }^{b)}$ Work performed in partial fulfillment of the requirements for the Ph. D. degree in Chemistry at the California Institute of Technology.

c) Contribution No, 5762 .
}

based upon theoretical or experimental considerations, ${ }^{10,11}$ may in part be attributed to these difficulties. These rules are predicated upon the validity of the "corresponding states" assumption. Here, some "universal function" may be used to predict the potential for a particular interaction, given appropriate distance and energy scaling parameters. ${ }^{10}$ The concept of such a universal functions has however, been abandoned in describing homonuclear interaction potentials, which could not formerly be obtained with sufficient accuracy. ${ }^{1 \mathrm{c}}$ As more data become available for the determination of heteronuclear interatomic potentials, it is increasingly apparent that flexible potentials are also needed to describe these interactions accurately. ${ }^{12}$ Indeed, in a recent computer simulation study ${ }^{13}$ hereafter referred to as Paper I, it was shown that the assumption of too rigid a mathematical form (e.g., having only two or three parameters) for the analysis of low-energy differential cross section (DCS) scattering data can introduce significant biases into the potentials extracted from such data. Such biases can be avoided only by using appropriately flexible mathematical forms in the analysis of the DCS data. Consequently, the use of excessively simple potentials and their combination rule progeny should be abandoned. It is important to use a more realistic approach to determine accurate heteronuclear potentials for simple van der Waals interactions.

In the present paper, we apply such an approach to the determination of the He-Ar potential, which has often served as a prototype for discussions of heteronuclear rare gas pair interactions. Experimental details for the acquisition of the DCS data presented in this study are described in Sec. II. The data reduction procedure used to analyze these DCS measurements are outlined in Sec. III. In Sec. IV we present our DCS data and several potentials obtained from them. 


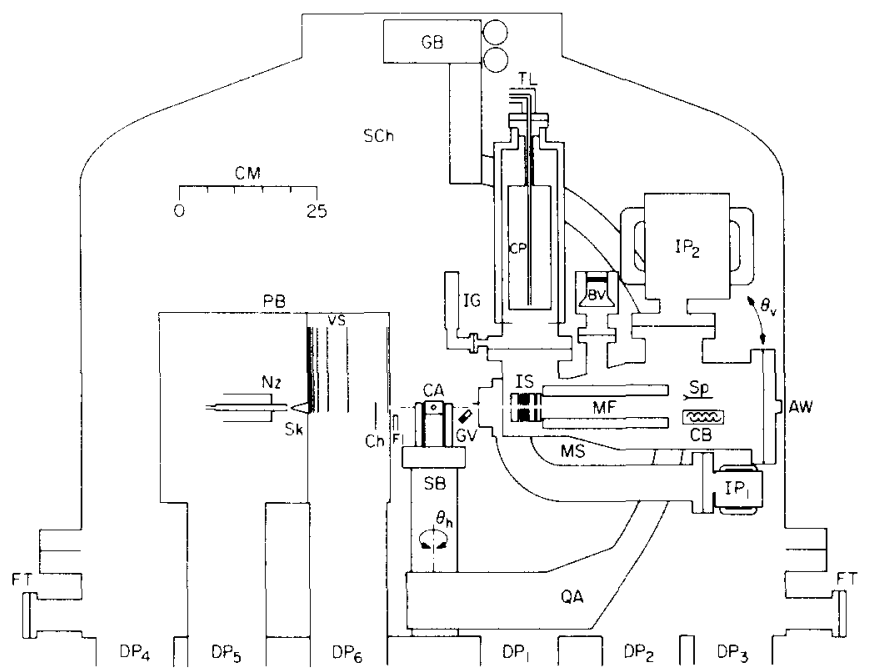

FIG. 1. Vertical cross section of the molecular beam apparatus, drawn approximately to scale. FT=copper gasket feedthroughs, $\mathrm{DP}_{1-5}$-oil diffusion pumps, $\mathrm{DP}_{6}=$ mercury diffusion pump, $\mathrm{PB}=$ primary beam source, $\mathrm{Nz}=$ nozzle, $\mathrm{Sk}=$ skimmer, $\mathrm{Ch}=$ beam chopper, VS=velocity selector, $\mathrm{FL}=$ beam flag, $\mathrm{SB}$ = secondary beam source, $\mathrm{CA}=$ capillary array, $\mathrm{SCh}=$ scattering chamber, $\mathrm{QA}=$ mass spectrometer quadrant arm, $\mathrm{GB}=$ gear box and pulleys, MS= mass spectrometer detector, $\mathrm{AW}=$ alignment window, $\mathrm{IP}_{1}=5 \mathrm{l} / \mathrm{sec}$ ion pump, $\mathrm{IP}_{2}=25 \mathrm{l} / \mathrm{sec}$ ion pump, $\mathrm{CP}=$ liquid He cryopump, $\mathrm{TL}=$ transfer line, $\mathrm{IG}=$ ionization gauge, $\mathrm{BV}=$ bakeout valve, $\mathrm{GV}=$ gate valve, IS = electron bom bardment ion source and ion optics, $M F=$ quadrupole mass fil ter, $\mathrm{CB}=\mathrm{Cu}-\mathrm{Be}$ dynode electron multiplier, $\mathrm{Sp}=$ Spiraltron electron multiplier, $\theta_{h}=$ in-plane scattering angle, $\theta_{v}=$ out-ofplane scattering angle.

By comparing one of these potentials (of the Morsespline-van der Waals form) with those obtained in two other laboratories, ${ }^{14,15}$ using the same mathematical form, we analyze in Sec. $V$ the equivalence of the DCS scattering data of the three laboratories. We also discuss in that section the appropriateness of the forms used in Sec. IV, and those used in previous studies, ${ }^{14-16}$ for accurately describing the interatomic potential. In Sec. VI we examine the consistency of our potentials with integral cross section scattering data, ${ }^{17,18}$ and use these to potentials to predict the zero-point dissociation energy of the bound $\mathrm{He}-\mathrm{Ar}$ diatom for which spectroscopic measurements are not yet available. ${ }^{19}$ We also adjust our $\mathrm{M}^{2} \mathrm{SV}^{20}$ and SPFD ${ }^{21,22}$ potentials for interatomic distances to which the DCS data are insensitive, ${ }^{13}$ to provide a modified potential appropriate for a description of equilibrium ${ }^{23-25}$ and of gas transport $\mathrm{t}^{26-29}$ properties. Finally, in Sec. VII, we present a concise characterization of the $\mathrm{He}-\mathrm{Ar}$ potential.

\section{EXPERIMENTAL}

The crossed molecular beam apparatus used in our laboratory is shown schematically in Figs. 1 and 2. The major components are a movable, differentially pumped mass spectrometer detector, a fully supersonic primary beam, and an intense but only slightly supersonic secondary beam. The present version of the ap- paratus is an extensive improvement over earlier ones, which have been described elsewhere. ${ }^{30,31}$

The two beam sources and detector support arc are mounted to the base of a 12001 vacuum chamber, as shown in Fig. 1. All internal components of the apparatus are serviced via feedthroughs (FT) placed around the base circumference. The upper part of the chamber is a stainless steel bell jar which may be lifted free of the internal components, allowing access for work on them. The main vacuum chamber is pumped by four liquid nitrogen $\left(\mathrm{LN}_{2}\right.$ ) trapped $25 \mathrm{~cm}$ oil diffusion pumps $\left(\mathrm{DP}_{1}\right.$ through $\mathrm{DP}_{4}$ ), with a combined measured pumping speed of $S=1600 \mathrm{l} / \mathrm{sec}$.

The primary beam (PB) is of the supersonic design, ${ }^{32}$ with nozzle $(\mathrm{Nz})$ and skimmer $(\mathrm{Sk})$ diameters of 0.07 and $0.64 \mathrm{~mm}$, respectively. The nozzle is fitted to the end of a gas inlet tube which, serving as a piston, is mounted to a motor-driven translator. With this arrangement, the nozzle-skimmer distance may be adjusted remotely, while maintaining an accurate beam alignment. The nozzle stagnation chamber is evacuated by a fifth $25 \mathrm{~cm}$ oil diffusion pump $\left(\mathrm{DP}_{5} ; S=400 \mathrm{l} / \mathrm{sec}\right)$; a second chamber downstream of the skimmer is differentially pumped by a $25 \mathrm{~cm} \mathrm{LN}_{2}$-trapped mercury diffusion pump $\left(\mathrm{DP}_{6} ; S=120 \mathrm{l} / \mathrm{sec}\right)$. This buffer chamber houses a mechanical beam chopper $(\mathrm{Ch})$ which modulates the primary beam, simultaneously generating a square wave signal with an infrared light-emitting diode and photocell arrangement. This signal is used as a reference input to a phase-sensitive lock-in detection system (see below). Also enclosed in the primary beam buffer chamber is a mechanical slotted-disk velocity selector (VS), ${ }^{33}$ used for beam velocity distribution measurements. The modulated primary beam, which may be interrupted by a pneumatically-operated beam flag (F1), emerges into the main (scattering) chamber through a collimator of $1.6 \mathrm{~mm}$ diameter. This final collimator $\left(C_{1}\right)$ is located $79 \mathrm{~mm}$ from the scattering center (SC), as depicted in Fig. 2.

Returning to Fig. 1, the secondary beam (SB) is

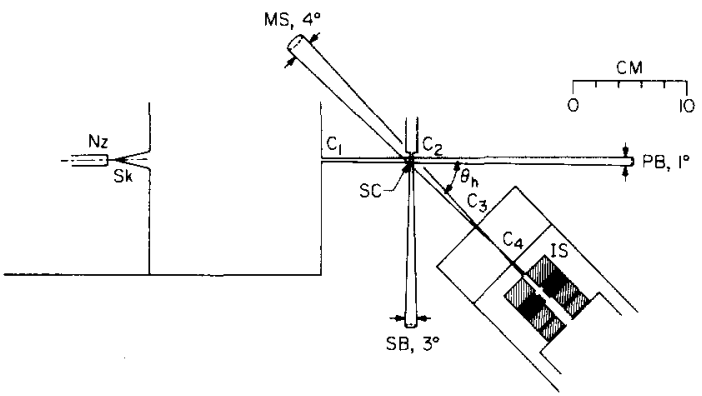

FIG. 2. Schematic of the crossed beam scattering zone, viewed from above and drawn to scale. SC=scattering center, PB = primary beam angular divergence cone, $\mathrm{SB}=$ secondary beam angular divergence cone, $\mathrm{MS}=$ detector penumbral ${ }^{39}$ viewing cone (umbral viewing cone angle $=0.7^{\circ}$ ), $C_{1}=$ primary beam collimator, $\mathrm{C}_{2}=$ secondary beam collimator, $\mathrm{C}_{3}=$ detector entrance collimator, $\mathrm{C}_{4}=$ detector buffer chamber collimator, $\mathrm{Nz}$ = primary beam nozzle, $\mathrm{Sk}$ = primary beam skimmer, IS = mass spectrometer ion source. 
shown as consisting of a glass capillary array $(\mathrm{CA}),^{36,37}$ through which the beam gas is admitted directly into the scattering chamber ( $\mathrm{SCh}$ ). The array is $0.61 \mathrm{~mm}$ thick with individual capillary diameters of $0.002 \mathrm{~mm}$, and has a transparency to gas flow of $50 \%$. The area of the array actually used is defined by a retaining cap with a hole of $1.6 \mathrm{~mm}$ diameter $\left(\mathrm{C}_{2}\right)$, located $6 \mathrm{~mm}$ from the SC (Fig. 2). The retaining cap also seals the array to the gas feed tube to prevent leakage around it. This assembly is mounted in a block which may be tilted upwards by remote activation of a pneumatic bellows, uncrossing the beams. Turning the secondary beam "off" in this fashion, the modulated background signal can be measured without affecting it as seriously as a beam flag would, since the secondary beam gas flow is uninterrupted. The bottom surface of the block is machined flat, which in mating to a similarly flat support structure, ensures precise alignment each time the beam is lowered to intersect the primary.

Although optimum operating conditions could be estimated theoretically for these beam sources, ${ }^{32,35}$ the approximations involved make it worthwhile, in addition to design calculations, to perform experimental optimization studies. For the primary beam, it was generally found in these studies that intensity maximization and velocity spread minimization (with respect to gas inlet pressure and nozzle-skimmer separation) were complementary characteristics, while the beam angular spread was independent of these variables. The primary beam nozzle-skimmer distance is optimized at $7 \mathrm{~mm}$; the differential chambers pumped by $\mathrm{DP}_{5}$ and $\mathrm{DP}_{6}$ are operated at pressures of $3 \times 10^{-4}$ and $2 \times 10^{-5}$ torr, respectively.

Optimization of the secondary beam on the other hand, required a compromise between intensity and angular divergence. It is also necessary to maintain a low scattering chamber pressure, as well as to avoid dimer formation (for some gases more condensable than Ar). Measured velocity distributions were found to be independent of the source pressure in the 2-6 torr range, and of the identity of the beam gas. It should be noted that because the secondary beam gas particles used in these experiments are heavier than those of the primary beam, and so do not contribute greatly to the relative velocity distribution, the narrowness of the secondary beam velocity distribution is much less crucial than that of the primary beam.

TABLE I. Beam operating conditions.

\begin{tabular}{llc}
\hline Characteristic & $\begin{array}{l}\text { Primary } \\
\text { beam }\end{array}$ & $\begin{array}{l}\text { Secondary } \\
\text { beam }\end{array}$ \\
\hline beam gas & $\mathrm{He}$ & $\mathrm{Ar}$ \\
inlet pressure/torr & 1300 & 3.2 \\
inlet temperature $/{ }^{\circ} \mathrm{K}$ & 298 & 298 \\
angular FWHM $^{\mathrm{a}} / \mathrm{deg}$ & 1 & 3 \\
most probable velocity $/(\mathrm{km} / \mathrm{sec})^{\text {velocity FWHM, }{ }^{\mathrm{a}} \Delta v / v}$ & 1.757 & 0.502 \\
Mach number $^{32}$ & 0.10 & 0.75 \\
\hline \hline
\end{tabular}

${ }^{\mathrm{a}}$ Full width at half-maximum.
The He-Ar scattering experiments described in this paper were performed using the optimized beam conditions presented in Table I. No primary beam velocity selection was used; the overall apparatus velocity resolution under these conditions is $11.6 \%$ FWHM. A measured primary beam attenuation of about $5 \%$, and a scattering chamber pressure below $3 \times 10^{-6}$ torr, are sufficiently low to ensure that the single-collision conditions essential for DCS measurements prevail.

The gas inlet system for the two beams have parallel needle leak valves and absolute pressure gauges. ${ }^{36}$ This arrangement ensures stable regulation and accurate monitoring of beam pressures over long periods of time. The secondary beam purity is periodically checked with the mass spectrometer detector, since although we analyze scattering events specifically for $\mathrm{He}$, we are unable to discriminate similarly against the identity of secondary beam particles.

Referring again to Fig. 1, we see that the detector may remotely be positioned at a variable angle in the plane of the beams by its supporting quadrant arm (QA), which rotates about a vertical axis passing through the SC. The center of this semicircular arm is coincident with the SC; the detector may also be moved along the rim of the QA, further permitting scans of scattering angles out of the plane of the beams. The force necessary to raise and lower the $120 \mathrm{~kg}$ bulk of the MS is transmitted through the vacuum wall via a rotary-motion feedthrough and a gear box (GB) with a 1000: 1 demultiplication ratio, using a cable and pulley arrangement. The in-plane and out-of-plane angular positions $\theta_{h}$ and $\theta_{v}$ are measured by angular resolving units ${ }^{37,38}$ geared to the vertical rotation axis of the $Q A$, and to the $Q A$ rim, respectively. The reproducibility of the position indicator system is $0.02^{\circ}$, and has a measured absolute accuracy of $\pm 0.05^{\circ}$. The detector angular range is restricted to $-20^{\circ}<\theta_{h}<100^{\circ}$ (measured from the primary beam towards the secondary) and $-12^{\circ}<\theta_{v}<23^{\circ}$ (upwards with respect to the plane of the beams).

Particles originating in the scattering region are detected by a separately enclosed quadrupole mass spectrometer (MS). As shown in Fig. 2, its entrance collimator of $1.6 \mathrm{~mm}$ diameter $\left(C_{3}\right)$, is located $80 \mathrm{~mm}$ from the SC. The scattered beam passes through an entrance buffer chamber, which is separated from the remainder of the MS enclosure by a second $1.6 \mathrm{~mm}$ diameter collimator $\left(\mathrm{C}_{4}\right)$ located $45 \mathrm{~mm}$ downstream of the first. The MS penumbral cone angle ${ }^{39}$ is thus $4^{\circ}$; its $7 \mathrm{~mm}$ diameter at the SC ensures that, regardless of the detector position, the entire scattering zone is observed. Note however, that the umbral cone angle ${ }^{36}$ is only $0.7^{\circ}$. The overall apparatus angular resolution is determined by the primary beam angular divergence and the size of the scattering volume, as well as by the detector's angular resolution. For a He primary beam, the effective overall angular resolution as determined in Sec. IV, has a FWHM of $1.9^{\circ}$.

Precise alignment of the apparatus is a prerequisite for good DCS measurements to be made. The principal alignment point, defined to be the location of the $\mathrm{SC}$, is 
at a specific height on the cylindrical symmetry axis of the scattering chamber. A precision optical surveyor's telescope, sighting through an alignment window (AW) set in a glass-to-metal seal at the rear of the MS enclosure (Fig. 1), is used to align the long axis of the MS. At $\theta_{h}=\theta_{v}=0^{\circ}$, the MS collimators $\left(C_{3}\right.$ and $\mathrm{C}_{4}$ of Fig. 2) are aligned with the tip of a pin placed exactly at the SC. The same MS axis is simultaneously adjusted to be parallel to the plane of the QA so that out-of-plane scans may be made with a constant $\theta_{h}$. The two beam axes are also optically aligned with the pin at the SC and are made to intersect at $90^{\circ}$.

Since the primary beam is relatively far from the $\mathrm{SC}$ and its location determines the zero position of the angular scattering scale, its precise alignment is especially important. With the MS at $\theta_{h}=\theta_{v}=0^{\circ}$, the two MS collimators, the pin at the SC, and the three primary beam apertures are all within $0.1 \mathrm{~mm}$ of collinearity, corresponding to an error of less than $0.1^{\circ}$ in either $\theta_{h}$ or $\theta_{v}$. To check the precision of detector rotation about the $\mathrm{SC}$, the distance between the entrance collimator of the MS and the pin at the SC is measured as a function of the detector position. The maximum deviation observed is $0.1 \mathrm{~mm}$ throughout the accessible angular range; the detector and primary beam misalignments are thus no more than $0.1^{\circ}$ each.

The MS entrance chamber is differentially pumped (Fig. 1) by a small ion pump $\left(\operatorname{IP}_{1} ; S=51 / \mathrm{sec}\right)$; the main MS chamber is pumped by a larger one $\left(\mathrm{IP}_{2} ; S=25\right.$ $1 / \mathrm{sec})$. These are supplemented during experiments by a liquid helium cryopump (CP), which is periodically filled via a vacuum-jacketed transfer line (TL). The cryopump is surrounded by an $\mathrm{LN}_{2}$-cooled radiation shield; its one liter capacity is sufficient for a holding time of about one hour under operating conditions, even though located directly above the MS ionizer. Serving as the cryogenic surface, the bottom of the dewar is an electropolished stainless steel disc of $75 \mathrm{~mm}$ diameter, and at low pressure ( $\approx 10^{-8}$ torr $)$ it is an efficient pump for all gases, including $\mathrm{Ne}, \mathrm{H}_{2}$, and even $\mathrm{He}$, with a conductance limit of $S=400 \mathrm{l} / \mathrm{sec}$. The pressure in the ionizer region during experiments is about $1 \times 10^{-9}$ torr as measured with an uncalibrated ionization gauge (IG).

All MS components are bakeable up to $250^{\circ} \mathrm{C}$ to ensure attainability of the very high vacuum necessary for these experiments. During bakeouts, the MS is evacuated only by the scattering chamber diffusion pumps via a remotely operated bakeout valve (BV). When a sufficiently low pressure is reached at the end of a bakeout, this bellows-activated valve is closed; a similarly operated gate valve (GV) is then also closed over the MS entrance collimator. These two valves thus serve to isolate the MS enclosure from the rest of the system; the former remains at very high vacuum even when the bell jar is vented for periodic maintenance.

After entering the main MS chamber, particles pass through a high-efficiency electron-impact ion source (IS), ${ }^{40,41}$ usually operated at $25 \mathrm{~mA}$ emission current. Ions thus produced are extracted and focused, then analyzed by a quadrupole mass filter (MF) ${ }^{41}$ and finally detected. Mass resolution and ion focusing parameters are adjusted to resolve adjacent mass peaks, while maintaining their symmetry and maximizing the signal at any chosen mass between 1 and $140 \mathrm{amu}$.

Ion detection and signal processing may be chosen in either an analogue or digital mode by controls outside the vacuum chamber. The analogue mode utilizes a 14stage $\mathrm{Cu}-\mathrm{Be}$ dynode electron multiplier (CB), whose output is passed immediately through a bakeable lowgain dc preamplifier ${ }^{41}$ located within the MS enclosure. This signal is then processed outside the vacuum chamber by an ac tuned preamplifier followed by a phasesensitive lock-in amplifier ${ }^{42}$ synchronized to the primary beam chopper reference signal. The modulated, in-phase component of the signal is thus extracted from a largely dc background, and is then smoothed by an $\mathrm{RC}$ integrator. The output is sent to an analogue-todigital (A-D) converter, which is sampled and averaged by a laboratory minicomputer.

The digital mode of detection, which was used for these experiments, utilizes a Spiraltron ${ }^{34}$ continuous dynode electron multiplier (Sp). The resulting narrow pulses ( $F W H M \approx 1 \mu \mathrm{sec}$ ) are passed through an amplifier-discriminator and are counted by a synchronous up-down counter. At the $160 \mathrm{~Hz}$ modulation frequency used, time-of-flight effects for a room-temperature He beam blur the edges of the modulated beam-on and beam-off time intervals by a phase equivalent of almost $1^{\circ}$. To avoid these distortions, we count only in the central $90 \%$ of these intervals after suitably phase shifting the reference signal. Care is also taken to operate the Spiraltron in the linear gain region below about $7 \times 10^{4}$ counts $/$ sec with a two-terminal mode voltage of $2.8 \mathrm{kV}$. Because of this restriction, it is necessary to lower the ionizer emission current to only $1 \mathrm{~mA}$ in order to take low-angle scattering data $\left(\theta_{h}\right.$ or $\theta_{v}$ ₹ $3.5^{\circ}$ ).

Using either the digital or analog modes, a raw data point at a particular $\theta_{h}$ or $\theta_{v}$ is obtained by accumulating the modulated signal with the secondary beam lowered to intersect the primary, then uncrossing the beams and subtracting the modulated signal for the same length of time. The average of three successive measurements is then normalized to a repetitively measured reference scattering signal (at $\theta_{k}=4.6^{\circ}$ or $\theta_{v}=4.9^{\circ}$ ). The entire DCS vs scattering angle curve is measured at least three times, a simple average being performed to yield the experimental DCS curve. Signal-to-noise $(S / N)$ ratios decrease from nearly 100 for low-angle scattering with total counting times of 3 min per angle, to about 10 at the widest angles used $\left(\sim 20^{\circ}\right)$, with counting times of $1 \mathrm{~h}$.

The experimental error for the individual data points is determined independently of the $S / N$ ratios quoted above, since the latter account only for statistical fluctuations of the individual measurements. Other sources of error include reference signal instability (std. dev. $\approx 5 \%)$ and some slight uncertainty $\left(<0.05^{\circ}\right)$ of the MS position. In a detailed set of experiments, the standard deviation of the scattered signal was measured as a 
function of scattering angle, and was fitted by a quadratic polynomial increasing monotonically over the angular range of these experiments. This polynomial was used thereafter to estimate the experimental error for each data point of the DCS curve.

Data around the first minimum of the $\operatorname{DCS}\left(\theta_{h}\right.$ or $\theta_{v}$ between $6.5^{\circ}$ and $8.5^{\circ}$ ) are also taken for the corresponding negative scattering angles. The zero positions of the angular scattering scale $\left(\theta_{0 h}\right.$ for in-plane and $\theta_{0 v}$ for out of plane) may then be determined by matching the position of this minimum as it appears on both sides of the primary beam, after making the appropriate laboratory to center-of-mass kinematic transformations. By plotting the first minimum on a scale accentuating the highly oscillatory structure of the DCS (see Sec. IV), we are able to locate the $\theta_{0}$ to within $0.03^{\circ}$ for both in-plane and out-of-plane scattering. Though small, these zero-angle corrections are necessary because of the great sensitivity of the DCS to the scattering angle. By comparison, the estimated accuracy of a beam centerline measurement from the intensity profile is $\simeq 0.2^{\circ}$. Angular scale corrections obtained in the above manner are less than $0.2^{\circ}$ relative to the scale established by the optical procedure, within the estimated accuracy of the latter. It was found however, that $\theta_{0}$ shifts of the order of $0.2^{\circ}$ produced detectable changes in the resulting interatomic potential, indicating that the higher accuracy of $0.03^{\circ}$ in determining $\theta_{0}$ is indeed necessary.

\section{DATA REDUCTION}

The data reduction procedure used in the course of this work has been carefully refined to distinguish between closely related interatomic pair potentials. This refinement required the calculation of accurate DCS's from assumed potentials, and accurate averaging over all experimental conditions.

Functionally, the procedure has three parts. First is an initialization used to set up the transformations from the center-of-mass (c.m.) frame to the particular laboratory frame of the experiment (scanning in the plane of the beams, or perpendicular to it with $\theta_{h}=0^{\circ}$ ). The second part uses the partial wave expansion to calculate monoenergetic DCS's in the c.m. from an assumed interatomic potential; transformations and appropriate apparatus parameters are then used to calculate an average lab DCS. Finally, the third part optimizes the parameters of the chosen potential form to obtain a least-squares best fit to the experimental DCS.

The initialization part consists of the following steps:

(1) Primary and secondary beam velocity distributions are calculated from the hypersonic expansion model $^{33}$ and the measured beam characteristics presented in Sec. II (see Table I). Between velocities corresponding to equal cut-off probabilities along these distributions, a number of Gaussian integration points are chosen independently for each beam, and are designated $v_{1 i}\left(i=1, \cdots, n_{1}\right)$ and $v_{2 j}\left(j=1, \cdots, n_{2}\right)$, respectively.
(2) We assume each of the beam angular divergences are described by cosine-squared distribution functions having the measured FWHM ${ }^{43}$ (Table I). From these the distribution of beam intersection angles is calculated, and a third set of Gaussian integration points, designated $\gamma_{k}\left(k=1, \cdots, n_{\gamma}\right)$, is chosen along this distribution.

(3) The beam intersection angle and velocity distributions are convoluted to calculate the distribution of relative collision velocities. Between relative velocities corresponding to equal cut-off probabilities, a fourth set of Gauss points, designated $u_{p}\left(p=1, \cdots, n_{u}\right)$, are chosen.

(4) For each of an input set of c.m. scattering angles, $\theta_{\text {c.m. }}$, we compute the corresponding laboratory scattering angles $\theta_{1 \text { ab }}^{i j k}\left(\theta_{\text {c.m. }}\right)$ using the c.m. - to-lab coordinate transformation specified uniquely by each $\left(v_{1 i}, v_{2 j}, \gamma_{k}\right)$ combination and the plane in which the detector moves. The corresponding Jacobian factors $J_{1 \mathrm{ab}}^{i j k}\left(\theta_{c, \mathrm{~m} .}\right)$ (appropriate for a number-density detector) and relative velocities $w_{i j k}$ are also calculated (see Appendix).

(5) The peaks of the $v_{1}, v_{2}$, and $\gamma$ distributions are used similarly to transform the input $\theta_{\text {c.m. }}$.'s to the appropriate lab frame. This additional set of lab angles, denoted $\theta_{1 \mathrm{ab}}^{\text {calc }}\left(\theta_{\text {c.m. }}\right)$, is used as the set at which the laboratory averaged DCS will be calculated in step 9 .

The quantities calculated in the initialization section above are stored for use throughout the second part, which follows:

(6) The $u_{p}$ of step 3 are used to calculate partial wave phase shifts from an assumed interaction potential (see Sec. IV for the potential forms considered). With our experimental conditions, and for a potential close to that of $\mathrm{He}-\mathrm{Ar}$, we find that JWKB phase shifts ${ }^{44}$ give slightly different (by about $4 \%$ or less) DCS's from those calculated using exact quantum mechanical (EQM) phase shifts. The latter were calculated by Numerov integration ${ }^{45}$ of the Schrödinger equation, and compare satisfactorily to tabulated phase shifts. ${ }^{46}$ Rosen-Yennie corrections ${ }^{47}$ may be applied to the JWKB phase shifts instead, resulting in highly accurate (better than $0.5 \%$ ) DCS's and substantial computational savings (see the end of this section). It is convenient to use these corrections only for smooth potentials because the numerical quadratures used require evaluations of second and third derivatives of the potential energy ${ }^{44,47}$; for any of the piecewise analytical potentials we use the EQM phase shifts.

(7) The partial wave summation ${ }^{48}$ is used to calculate DCS's at the $\theta_{c . m .}$ 's for each of the $u_{p}$ 's. DCS's at the $w_{i j k}$ relative velocities of step 4 are then obtained by interpolation over the $u_{p}$ 's, yielding the c.m. cross sections $\sigma_{c, m}^{i j k}\left(\theta_{c, m}\right)$.

(8) Unlike the $u_{p}^{\prime} \mathrm{s}$, the $w_{i j k}$ 's correspond to a particular combination of $v_{1 i}, v_{2 j}$, and $\gamma_{k}$, so that transformations to the lab frame may now be made. For each $i j k$ combination, we simply multiply the $\sigma_{\text {c.m. }}^{i j k}\left(\theta_{\text {c.m. }}\right)$ by the $J_{1 \mathrm{ab}}^{i j k}\left(\theta_{\text {c.m. }}\right)$ intensity factors of step 4 , to obtain 
the lab DCS's at the $\theta_{1 \mathrm{ab}}^{i j k}\left(\theta_{\text {c.m. }}\right)$ 's. For each $\left(v_{1 i}, v_{2 j}, \gamma_{k}\right)$ combination, this furnishes the function $\sigma_{1 \mathrm{ab}}^{\mathrm{ijk}}\left(\theta_{1 \mathrm{ab}}^{i j k}\right)$.

(9) Since the $\theta_{1 \mathrm{ab}}^{i j k}$ sets of angles are different for each $\left(v_{1 i}, v_{2 j}, \gamma_{k}\right)$ combination, we use the functions obtained in step 8 to calculate, by interpolation, the $\sigma_{1 \mathrm{ab}}^{i j k}\left(\theta_{1 \mathrm{ab}}^{\mathrm{calc}}\right)$ at the set of lab angles computed in step 5. The velocity and beam angular divergence averaged lab DCS can now be obtained by performing a Gaussian integration at each of the $\theta_{1 \mathrm{ab}}^{\mathrm{calc}}$ 's to obtain the $\sigma_{1 \mathrm{ab}}\left(\theta_{1 \mathrm{ab}}^{\mathrm{cal}}\right)$ 's.

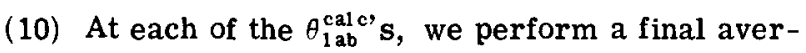
aging over the overall apparatus angular resolution. This averaging effect takes into account the divergence of the primary beam (independently of its effect on the beam intersection angle distribution of step 2 above) and the finite size of the scattering volume, in addition to the detector angular resolution. Since the correct overall resolution function would be very difficult to ascertain directly from apparatus considerations, its angular FWHM is treated as an adjustable parameter. The resolution function is assumed to have a cosinesquared shape; the suitability of this assumption is shown by noting that using a hyperbolic secant shape instead affects the fitted FWHM but not the calculated DCS. ${ }^{43}$ The earlier assumption of cosine-squared angular distributions for the primary and secondary beams (step 2 above) is now seen to be justified because of their significant contributions to the overall apparatus angular resolution. The treatment of the latter's FWHM as a fitted parameter is discussed in Sec. IV.

(11) The calculated scattering intensity at the experimental angles (the $\theta_{\text {exp }}$ 's) is obtained by interpolation from the averaged lab DCS's of step 10 . Since the measured DCS is arbitrarily normalized (i.e., no absolute intensity calibrations are made), the calculated DCS must be scaled to it. The scaling factor $\alpha$ is adjusted so that the quantity,

$$
\chi^{2}=\sum_{i=1}^{n} g_{i}\left(I_{i}-\alpha \sigma_{i}\right)^{2},
$$

is minimized with respect to $\alpha$. At each of the $n$ scattering angles, the $I_{i}$ are the measured (relative) cross sections, while the $\sigma_{i}$ are the calculated (absolute) cross sections. The normalized weighting factors $g_{i}$, are obtained at each data point from the associated experimental standard deviations $s_{i}$, using the expres$\operatorname{sion}^{49}$

$$
g_{i}=\frac{n s_{i}^{-2}}{\sum_{i=1}^{n} s_{i}^{-2}} .
$$

Following Eq. (10) of Paper I, we define a goodnessof-fit statistical index more suitable than $\chi^{2}$,

$$
\frac{\Delta \alpha_{q}}{\alpha}=\frac{1}{\alpha} t_{1-q}(n-k)\left[\frac{\chi^{2}}{(n-k) \cdot \sum_{i=1}^{n} g_{i} \sigma_{i}^{2}}\right]^{1 / 2} \text {. }
$$

Here $t_{1-q}(n-k)$ is Student's $t$-distribution statistic for a confidence level $q$ of the scaling factor $\alpha$, with $n$ data points and $k$ fitted parameters. ${ }^{49}$

(12) The final part of the data reduction procedure consists of using a weighted least-squares iterative scheme to optimize the parameters of a particular potential form. Our scheme is adapted from a general nonlinear regression algorithm proposed by Marquardt. ${ }^{50}$ We calculate the finite-difference derivatives of the DCS, with respect to each fitted potential parameter, by changing that parameter by a small amount (1\%) in only one direction, rather than by calculating the numerical derivatives symmetrically about the initial value of each parameter. This halves the computational effort and results in no loss of accuracy, because only an estimate of the first derivative is necessary. ${ }^{50}$ In one iterative cycle, steps 6-11 are repeated for each potential parameter being varied, and corrections are calculated with either the linearized $(\lambda=0$ in Marquardt's notation) or nonlinearized model. As indicated in Paper I, we find it best to use $\lambda=0.1$ or $\lambda=0.5$ for fits to potentials with four or more adjustable parameters; otherwise $\lambda=0$ may be used, resulting in faster convergence. ${ }^{13}$

Tuning of the integration and cut-off parameters to achieve computational accuracy and economy was performed. For these calculations a LJ12-6 potential, with $r_{m}$ and $\epsilon$ parameters close to those of $\mathrm{He}-\mathrm{Ar}$, were used to calculate accurate DCS's in the c. $m$. frame. These were extensively averaged and transformed to the lab frame using our experimental conditions. This DCS was then used as an input set of "data" to which other DCS's, calculated using the same potential form but with less accurate integration procedures, could be compared. To successfully fit the input DCS, and obtain the original $r_{m}$ and $\epsilon$ values, we found it necessary to use RY or EQM phase shifts in step 6, accurate to $0.001 \mathrm{rad}$, up to about $l=50$. For higher angular momenta, the JWKB phase shifts, accurate to $0.002 \mathrm{rad}$, were sufficient; the partial wave summation was truncated at a value of $l$ (of about 150) for which the corresponding phase shift was less than $0.001 \mathrm{rad}$. The DCS's in the c.m. frame were calculated in step 7 at $\theta_{\text {c.m.' }}$ 's spaced by $0.6^{\circ}$, at each of six Gauss-point c. m. velocities. It was also necessary to use a total of $144 i j k$ combinations along the beam velocity and intersection angle distributions to accurately simulate the c.m. - to-lab transformations and averagings of step 8. Recovery of the original LJ12-6 $r_{m}$ and $\epsilon$ values (to within $0.02 \%$ and $0.4 \%$, respectively) was realized, and essentially perfect fits were obtained $\left(\Delta \alpha_{0.95} / \alpha\right.$ $=0.18 \%$ ) with these calculational parameters. Less accurate lab DCS's gave poorer fits and/or inaccurate parameter values. These optimized calculational parameters were used for all the data reduction reported here, as well as for the fits reported in Paper I. Calculation of one fully averaged lab DCS curve requires about $30 \mathrm{sec}$ of IBM $370 / 158$ computer time using RY phase shifts, or about 100 sec using the EQM phase shifts.

\section{RESULTS AND DATA ANALYSIS}

As indicated at the end of Sec. II, small zero-angle corrections $\theta_{0 h}$ and $\theta_{0 v}$ must be applied to the in-plane and out-of-plane angular scales, respectively, before 


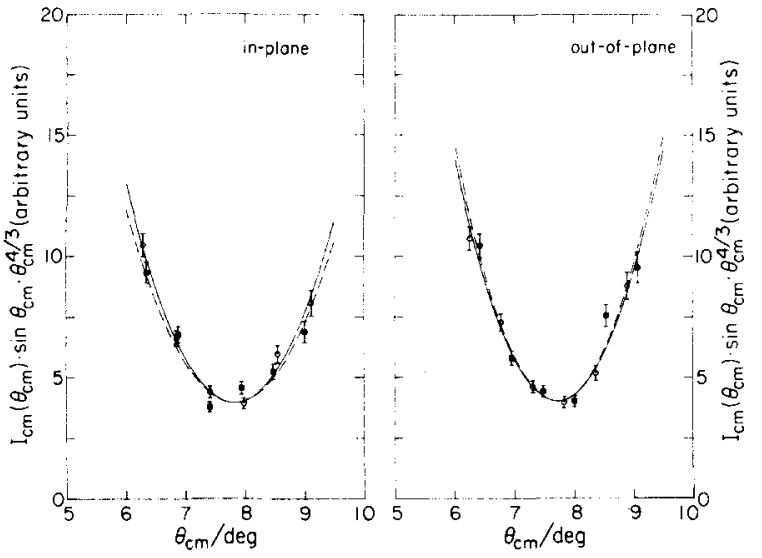

FIG. 3. He + Ar experimental cross sections in the vicinity of the first diffraction oscillation. $I_{\text {c.m. }}\left(\theta_{\text {c.m. }}\right)$ is the DCS in arbitrary units, transformed to the c.m. coordinate frame at the scattering angle $\theta_{\text {c.m. }}$. In-plane measurements on the left panel and out-of-plane measurements on the right panel are shown as points with error bars; open circles are for measurements at positive scattering angles and full circles are for negative scattering angles (in the sense defined in Sec. II). The lines are curve fits, quadratic in $\theta_{\text {c.m. }}$, used to locate the minimum position; solid lines are for positive scattering angles and dashed lines are for negative scattering angles.

the potential may be fit to the experimental DCS's. After fixed-velocity kinematic transformations to the c. $m$. coordiante system, in-plane and out-of-plane data taken in the vicinity of the first minimum on both sides of the primary beam are plotted in Fig. 3 on a scale accentuating the oscillatory structure of the DCS's. ${ }^{51}$ These data are least-squares fitted to second- or third-order polynomials in the scattering angle and the minima are located. The second-order fits are shown as the curves in Fig. 3; the third-order fits are quite similar and are not displayed. The in-plane (out-of-plane) zero-angle correction $\theta_{0 h}\left(\theta_{0 v}\right)$, is then found by iteratively adjusting the origin of the angular scale until the in-plane (out-of-plane) positions of the first minimum are the same for scattering on both sides of the primary beam. Zero-angle corrections obtained by fitting to the second- and third-order polynomials differ by $<0.05^{\circ}$; their averages are $\theta_{0 h}=+0.13^{\circ}$ and $\theta_{0 v}=-0.12^{\circ}$ relative to the optical zero.

Preliminary fits were made to the DCS's using the LJ12-6 potential form. In these fits, the overall apparatus angular resolution function's FWHM was treated as an adjustable parameter, which was fitted simultaneously with the $r_{m}$ and $\epsilon$ parameters. Best-fit values for the FWHM were $1.8_{3}^{\circ}$ and $1.8_{4}^{\circ}$ for the in-plane and out-of-plane fits, respectively. To preclude the possibility that these values of the fitted FWHM might depend on the potential form used, we also employed the SPFD form [Eq. (8) below with $N=2$, fitting $r_{m}, \epsilon$, $c_{6}$, and $b_{0}$ parameters as well as the FWHM] to fit the DCS's and obtained corresponding values $1.8_{0}^{\circ}$ and $1.9_{3}^{\circ}$. For all subsequent in-plane and out-of-plane data reductions, we fixed the overall apparatus angular resolution function's FWHM at $1.9^{\circ}$. This value is consistent with the apparatus geometry, as discussed towards the middle of Sec. II.
The differential cross sections (DCS's) for $\mathrm{He}+\mathrm{Ar}$ scattering at a most probable relative collision energy of $64.2 \mathrm{meV}$ using both in-plane (with $\theta_{v}$ set at $0^{\circ}$ ) and out-of plane (with $\theta_{h}$ set at $0^{\circ}$ ) motions of the detector were measured as described in Sec. II. Experimental DCS's were fit using the weighted least-squares data reduction procedure of Sec. III, in conjunction with the interatomic potentials described below. These potentials are written in reduced form as

$$
f(\rho)=\frac{V(r)}{\epsilon}, \rho=\frac{r}{r_{m}} ;
$$

$r_{m}$ is the radius of the van der Waals attractive minimum and $\epsilon$ is its depth. The zero of the potential, occurring at $r=\sigma$, may be related to $r_{m}$ and the potential shape $f(\rho)$. The potential shapes we consider are:

(a) Exponential-6 (exp-6), ${ }^{10}$

$$
f(\rho)=\frac{1}{\alpha-6}\left[6 e^{\alpha(1-\rho)}-\alpha \rho^{-6}\right] .
$$

This has the single shape parameter $\alpha$ which governs the behavior of the exponential repulsive term; note that the coefficient of the long-range attraction term is determined by $\alpha$.

(b) Morse-spline-van der Waals (MSV), ${ }^{52}$

$$
\left.\begin{array}{lc}
f(\rho)=e^{B(1-\rho)}\left[e^{\beta(1-\rho)}-2\right] & \text { for } \rho \leqslant \rho_{1} \\
f(\rho)=\left(\rho_{2}-\rho\right)\left[s_{1}\left(\rho_{2}-\rho\right)^{2}+s_{3}\right]+\left(\rho-\rho_{1}\right)\left[s_{2}\left(\rho-\rho_{1}\right)^{2}+s_{4}\right] \\
f(\rho)=-\sum_{i=1}^{3} c_{2 i+4} \rho^{-(2 i+4)} & \text { for } \rho_{1}<\rho<\rho_{2}
\end{array}\right\}
$$

The cubic spline coefficients $s_{i}(i=1-4)$ are fixed by smoothness conditions at the spline points $\rho_{1}$ and $\rho_{2}$, with the first one being chosen as $\rho_{1}=1+\beta^{-1} \ln 2$ (the inflection point of the Morse function). We usually introduce the multipole expansion at $\rho_{2}=1.6$; the $c_{i}$ coefficients are obtained from theoretical calculations. ${ }^{53,54}$ The MSV form thus has only the Morse $\beta$ parameter remaining to simultaneously control the shape of the attractive well and repulsive wall. Even the ESMSV form ${ }^{55}$ still has only a single parameter for the well and weakly repulsive regions.

(c) Morse-Morse-spline-van der Waals $\left(\mathrm{M}^{2} \mathrm{SV}\right) .{ }^{20}$

To avoid the inflexibility of the MSV form just mentioned, we use a second Morse function for $r<\sigma$, joined smoothly to the first one:

$$
\left.\begin{array}{lc}
f(\rho)=\frac{1}{\omega} e^{\left(\beta^{\prime}-\rho \beta \omega\right)}\left[e^{\left(\beta^{\prime}-\rho \beta \omega\right)}-2\right] & \text { for } \omega=\frac{\beta^{\prime}-\ln 2}{\beta-\ln 2} \text { and } \\
\rho<1-\beta^{-1} \ln 2 \\
f(\rho)=e^{\beta(1-\rho)}\left[e^{\beta(1-\rho)}-2\right] & \text { for } 1-\beta^{-1} \ln 2 \leqslant \rho \leqslant \rho_{1} \\
f(\rho)=\left(\rho_{2}-\rho\right)\left[s_{1}\left(\rho_{2}-\rho\right)^{2}+s_{3}\right] & \left(\rho-\rho_{1}\right)\left[s_{2}\left(\rho-\rho_{1}\right)^{2}+s_{4}\right] \\
f(\rho)=-\sum_{i=1}^{3} c_{2 i+4} \rho^{-(2 i+4)} & \text { for } \rho_{1}<\rho<\rho_{2}
\end{array}\right\}
$$


Having the additional shape parameter $\beta^{\prime}$, the $\mathrm{M}^{2} \mathrm{SV}$ potential permits a description of the repulsive wall independently from the attractive well; all other parameters have the same meanings as in the corresponding MSV potential.

(d) SPF-Dunham (SPFD), ${ }^{21,22}$

$$
\left.\begin{array}{ll}
f(\rho)=b_{0} \lambda^{2}\left(1+\sum_{i=1}^{N} b_{i} \lambda^{i}\right)-1 & \text { for } \lambda=1-1 / \rho \\
& \text { and } \rho<\rho_{f} \\
f(\rho)=-\sum_{i=1}^{3} c_{2 i+4} \rho^{-(2 i+4)} & \text { for } \rho \geqslant \rho_{f} .
\end{array}\right\}
$$

The two highest-order SPFD coefficients $b_{N}$ and $b_{N-1}$ are fixed by smoothness conditions at $\rho=\rho_{f}$. As for the MSV and $M^{2} S V$ potentials, the multipole expansion using theoretical $c_{i}$ coefficients is normally used in this paper beyond $\rho_{f}=1.6$; results for the influence of other $\rho_{f}$ values are presented in Sec. VI. Our usual choice of $N=3^{13}$ thus leaves the $b_{0}$ and $b_{1}$ parameters to determine the shape of the repulsive wall and attractive well regions of the potential.

For reduced distances below $\rho=0.65$, the $\exp -6$ and SPFD potentials often show spurious maxima or oscillations. These are removed by using a Born-Mayer exponentially repulsive wall, i. e., $f(\rho)=A e^{-b \rho}$, from some joining point $\rho_{i}$ downwards. Smoothness conditions at $\rho_{i}$ are used to fix $A$ and $b$. Trial calculations show that values of $\rho_{i}<0.75$ have no effect on the scattering results and we usually use $\rho_{i}=0.70$; also see Sec. VI.

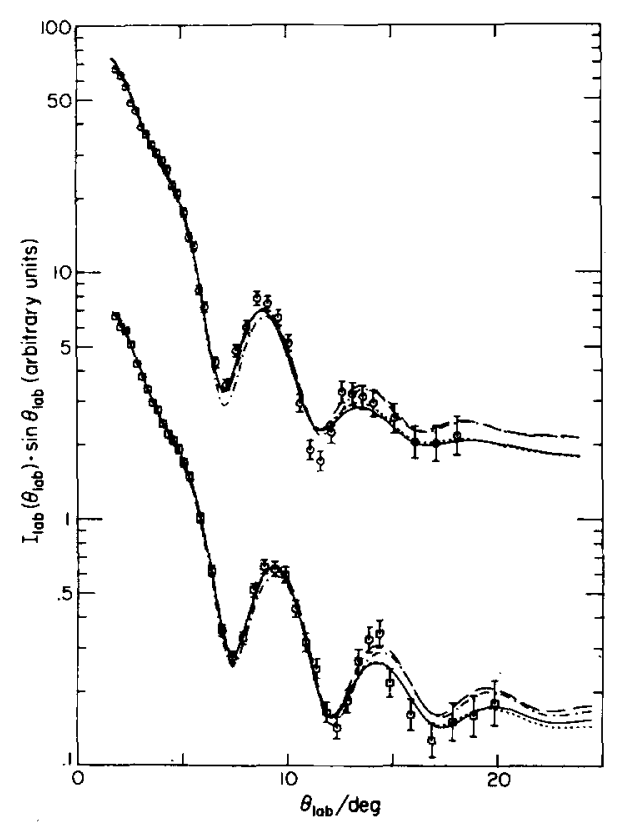

FIG. 4. Laboratory differential cross sections for in-plane and out-of-plane scattering; the latter are shifted downwards by one decade for clarity of display. Points with error bars are experimental measurements of the $\mathrm{He}+\mathrm{Ar}$ differential scattering cross sections $I_{1 \mathrm{ab}}\left(\theta_{1 \mathrm{ab}}\right) \cdot \sin \theta_{1 \mathrm{ab}}$ vs $\theta_{1 \mathrm{ab}}$ at an average relative collision energy of $64.2 \mathrm{meV}$. Curves are DCS's calculated from potentials fit separately to the two sets of experimental data. Solid line: SPFD potential; dotted line: $\mathrm{M}^{2} \mathrm{SV}$ potential; dashed line: MSV potential; dash-dotted line: exp-6 potential. The potential parameters are given in Table II.

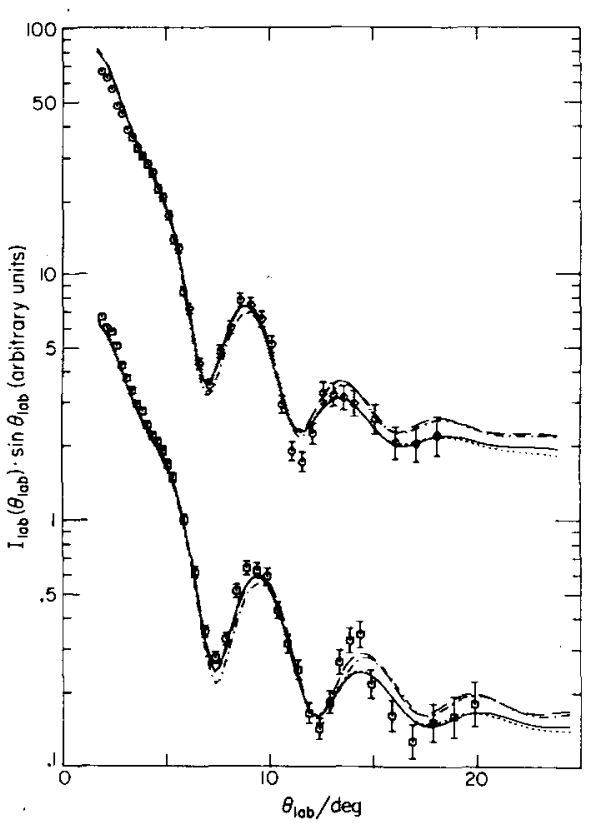

FIG. 5. Laboratory differential cross sections for in-plane and out-of-plane scattering. Curves are DCS's calculated from potentials fit to the combined sets of experimental data (parameters given in Table II); other symbols as in Fig. 4.

The choice of these four representations for the potential energy function was dictated by the computer simulation results of Paper I. In this investigation of the suitability of different potential energy forms $f(\rho)$ for determining accurate interatomic potentials from regression analyses of DCS measurements, ${ }^{56,57}$ it was found that the most successful potentials had at least four fitted parameters. Different parameterizations for different regions of the potential were generally helpful in avoiding high statistical parameter correlations, allowing meaningful parameter values to be obtained. With fits including only the $c_{6} \rho^{-6}$ term for the long-range region of the potential, up to five independent potential parameters (including $c_{B}, r_{m}$, and $\epsilon)$ could justifiably be determined. The $\mathrm{M}^{2} \mathrm{SV}$ and SPFD (with $N=3$ ) potentials of Eqs. (7) and (8), respectively, were found to be the most satisfactory in recovering the "accurate" interatomic potentials of the simulation study. ${ }^{13}$ Note that since we use the the oretical $^{54}$ long-range dispersion interaction in fitting the experimental DCS's (which omits $c_{6}$ from the set of five adjusted parameters), these two potentials have the maximum number of parameters that may justifiably and reliably be determined from our DCS measurements, for the attractive well and weakly repulsive regions of the potential. The exp- 6 and MSV forms of Eqs. (5) and (6), respectively, were included in our analysis for a comparison with potentials previously obtained from DCS measurements, ${ }^{14-16}$ although the simulation results showed that they are somewhat less satisfactory representations of the potential energy (especially the exp-6 form). ${ }^{23}$

The in-plane and out-of-plane experimental DCS's, with $2^{\circ}<\theta_{h}<17^{\circ}$ and $2^{\circ}<\theta_{v}<20^{\circ}$, respectively, are shown in Figs. 4 and 5. The curve fits of Fig. 4 were 
TABLE II. Interatomic potential fits to the experimental DCS's.

\begin{tabular}{|c|c|c|c|c|c|c|}
\hline Potential type $^{\mathrm{a}}$ & $\begin{array}{l}\text { scattering } \\
\text { frame }^{b}\end{array}$ & $r_{m} / \AA$ & $\epsilon / \mathrm{meV}$ & shape parameters $\mathrm{s}^{\mathrm{C}}$ & $x^{2 \mathrm{~d}, \mathrm{e}}$ & $\Delta \alpha_{0.95} / \alpha^{\theta}(\%)$ \\
\hline \multirow[t]{3}{*}{ exp-6 } & in-plane & 3.51 & 1.92 & $\alpha=11.2$ & $1300(3)$ & 2.89 \\
\hline & out-of-plane & 3.95 & 2.27 & 12.3 & $650(3)$ & 1.97 \\
\hline & combined & $3.53_{0}$ & 2.06 & 11.6 & $3900(3)$ & 2.40 \\
\hline \multirow[t]{3}{*}{ MSV } & in-plane & 3.57 & 2.01 & $\beta=5.01$ & $930(3)$ & 2.46 \\
\hline & out-of-plane & 3.58 & 2.36 & 5.61 & $460(3)$ & 1.65 \\
\hline & combined & $3.57_{2}$ & 2.16 & 5.26 & $3300(3)$ & 2.19 \\
\hline \multirow[t]{3}{*}{$\mathrm{M}^{2} \mathrm{SV}$} & in-plane & 3.67 & 1.66 & $\beta=4.20 ; \beta^{\prime}=7.70$ & $740(4)$ & 2.22 \\
\hline & out-of-plane & 3.64 & 2.01 & $\begin{array}{ll}4.73 & 9.78\end{array}$ & $270(4)$ & 1.29 \\
\hline & combined $^{f}$ & $3.65_{g}$ & 1.80 & 4.41 & $2900(4)$ & 2.08 \\
\hline \multirow[t]{3}{*}{$\operatorname{SPFD}(N=3)$} & in-plane & 3.63 & 1.53 & $b_{0}=11.5 ; b_{1}=-3.91$ & $700(4)$ & 2.17 \\
\hline & out-of-plane & 3.55 & 2.00 & $24.0 ; \quad-5.33$ & $270(4)$ & 1.29 \\
\hline & combined $^{q}$ & $3.57_{3}$ & 1.76 & -4.69 & $2900(4)$ & 2.07 \\
\hline
\end{tabular}

"Defined in Eqs. (5) through (8) of the text...

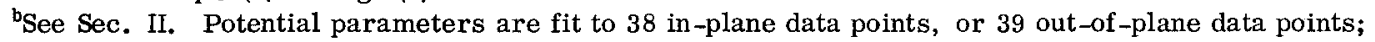
the combined potentials are fit to all 77 data points.

'Symbols have the same meaning as in Eqs. (5) through (8) of the text. The MSV and $\mathrm{M}^{2} \mathrm{SV}$ potentials also have $\rho_{2}=1.60$; the SPFD potential has $\rho_{f}=1.60$. These three potentials use the theoretical dispersion constants ${ }^{53,54} C_{6}=5.867 \mathrm{eV} \AA^{6} ; C_{8}=25.68 \mathrm{eV} \AA^{8} ; C_{10}=152.1 \mathrm{eV} \AA^{10}$.

${ }^{d}$ Scattering intensity normalized to 500 at $\theta_{h}=0.0^{\circ}, \theta_{v}=4.9^{\circ}$, and to 465 at $\theta_{h}=4.6^{\circ}, \theta_{v}=0.0^{\circ}$ for the in-plane fits; the number of parameters varied is given in parentheses.

${ }^{\circ}$ See Eqs. (1) and (3).

${ }^{\mathrm{f}}$ Best potentials.

obtained by treating the in-plane and out-of-plane data sets independently of one another (i.e., using separate potentials to fit them), while those of Fig. 5 were obtained by combining these data sets (and fitting a single potential to them). For each of the four potential models considered, three different potentials are thus extracted from the data. The respective potential parameters are shown in Table II, and the corresponding $M^{2} S V$ and SPFD potentials are displayed in Fig. 6 . It should be noted that in combining the data sets, the inplane scattering intensity at the reference angle (see the end of Sec. II) was measured carefully with respect to the out-of-plane reference intensity (standard deviation $\simeq 3.5 \%$ ). This permits the same factor $\alpha$ of $\mathrm{Eq}$. (1) to be used in scaling the in-plane and out-of-plane calculated DCS's to the corresponding experimental data (see the end of Sec. III).

It may be seen from Fig. 6 that the potential obtained by simultaneous fitting to both the in-plane and out-ofplane DCS's (termed the "combined potential") is very nearly the average of the potentials obtained by separate fits to these DCS's (termed the "in-plane potential" and the "out-of-plane potential", respectively). In view of the simulation results of Paper $I$, we expect the average, and therefore the combined potential, to be more accurate than either of the others. ${ }^{58}$ The differences between the in-plane or out-of-plane potentials and the more accurate combined potential are within the error bars quoted in Paper I for the "attractive well region" as defined in that paper. Of course, this is also true of the "attractive tail region" where the potentials are constrained to be identical. ${ }^{54}$ However, the $\pm 40 \%$ discrepancy in the "repulsive wall region" (see Fig. 6) is beyond the error bar of $\pm 30 \%$ established in Paper I for $r \gtrless 3.1 \AA$; this may be caused by several factors. Actual experimental data scatter may be larger than the error bars used in Paper I and depicted in Figs. 4 and 5 . The effect may be the most severe at the larger angles where the signal level is lowest (note for example, the sharp drop in the out-of-plane DCS at $\theta$ $=15^{\circ}$ ); we would then expect to have a poorer determin-

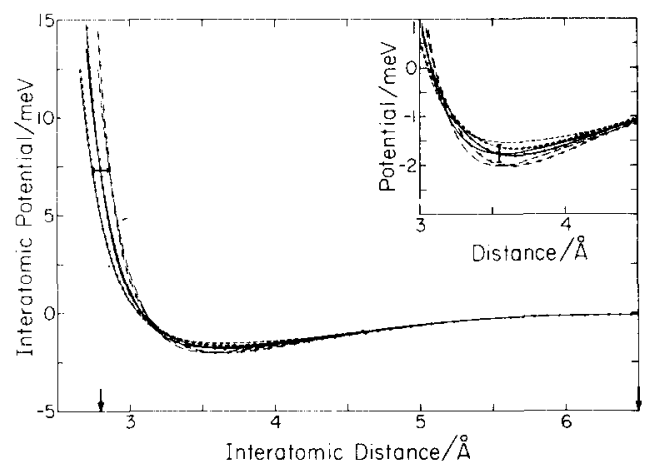

FIG. 6. Interatomic potentials for He-Ar as a function of interatomic distance. The inset magnifies the vertical scale of the potential energy minimum region by a factor of 2.5 . Curves with dots are $\mathrm{M}^{2} \mathrm{SV}$ potentials; those without are SPFD potentials. Solid curves: fits to the combined in-plane and out-ofplane data sets (best potentials); short dashed curves: fits to the in-plane data only; long dashed curves: fits to the out-ofplane data only. The vertical error bar in the inset indicates the estimated inaccuracy of $\pm 10 \%$ in our determination at the minimum, while the horizontal error bar at $7.5 \mathrm{meV}$ on the repulsive wall indicates the distance equivalent to the $\pm 30 \%$ uncertainty of the potential there. The arrows on the abscissa at interatomic distances of 2.8 and $6.5 \AA$ delineate the range of sensitivity of the room-temperature experiments to the DCS. ${ }^{13}$ 
ation of the potential primarily in the repulsive region. Possibly there also exists some systematic errors in the apparatus relating to the in-plane or out-of-plane detector motion; data reduction of the combined DCS's was performed to eliminate the effects of such systematic errors in determing the more accurate combined potentials. Consequently, only the combined potentials will be discussed hereafter; error bars claimed for them may be widened slightly beyond those quoted in Paper I.

\section{DISCUSSION}

On the basis of the computer simulation results of Paper I, we have selected the $\mathrm{M}^{2} \mathrm{SV}$ [Eq. (7)] and thirdorder SPFD $[N=3$ in Eq. (8)] potentials as the most suitable ones for analyzing the present DCS data. ${ }^{13}$ Our best potentials (the "combined potentials" of the previous section) can now be compared with others that have been obtained from fits either entirely or primarily to DCS scattering data. These are the exponentialspline-MSV (ESMSV), ${ }^{14}$ the exp-6, ${ }^{16}$ and the HartreeFock-Dispersion (HFD) ${ }^{15}$ potentials. A large variety of other potentials have been obtained from fits to only transport or gas-imperfection properties (see Ref. 14 for a recent compendium of these potentials). We begin with a discussion of reasons to avoid comparing potentials obtained from these different types of experiments (see also Sec. VI).

The second virial coefficient is given classically by ${ }^{10}$

$$
B(T)=2 \pi N_{\mathrm{Av}} r_{m}^{3} \int_{0}^{\infty}\left[1-e^{-\epsilon f(\rho) / k T}\right] \rho^{2} d \rho,
$$

where $T$ is the temperature, $N_{A v}$ is Avogadro's number, $k$ is the Boltzmann constant, and $r_{m}, \epsilon$, and $f(\rho)$ have the meanings introduced in Eq. (4). For $\mathrm{He}-\mathrm{Ar}$ at $T$ $>200^{\circ} \mathrm{K}$, quantum mechanical corrections ${ }^{10}$ are small (<5\%), so that the sensitivity of $B(T)$ data to different regions of the potential may be gauged by plotting the integrand of Eq. (9) as a function of $\rho$, as shown in Fig. 7. We may change the repulsive region without affecting the rest of the potential by altering the inner joining point $\rho_{i}$, where a Born-Mayer exponentially repulsive wall is joined smoothly to the SPFD potential (see Sec. IV). At $T=600^{\circ} \mathrm{K}$, the effect on the integrand of changing $\rho_{i}$ from 0.80 to 0.81 (see Sec. VI) is seen to be significant (though small) for reduced distances $\rho>0.65$; we conclude that $B(T)$ data are sensitive to the repulsive region of the interatomic potential in the range $0.65 \approx$ $\rho \approx 0.86$ [note that $f(\rho)=0$ at $\rho=0.862$ ]. At $T=200^{\circ} \mathrm{K}$, a similar argument indicates sensitivity in the range $0.69 \approx \rho \approx 0.86$; in both cases the lower limit may easily be determined by noting the minimum distance for which the integrand deviates noticeably from a simple $\rho^{2}$ dependence. Consequently, in considering the relative contributions to $B(T)$ in the repulsive $(\rho ₹ 0.86)$ and attractive $\left(\rho \Sigma_{0} .86\right)$ regions of the potential, we are concerned only with the integrand beyond $\rho=0.65$ at $T$ $=600^{\circ} \mathrm{K}$, or beyond $\rho=0.69$ at $T=200^{\circ} \mathrm{K}$. It is then seen from Fig. 7 that the shallowness of the $\mathrm{He}-\mathrm{Ar}$ attractive well causes $B(T)$ data to be as sensitive to the attractive region as to the repulsive wall only for low temperatures $\left(T \ll 200^{\circ} \mathrm{K}\right)$. We will therefore follow the precedent of Chen et al. ${ }^{14}$ who found that they could

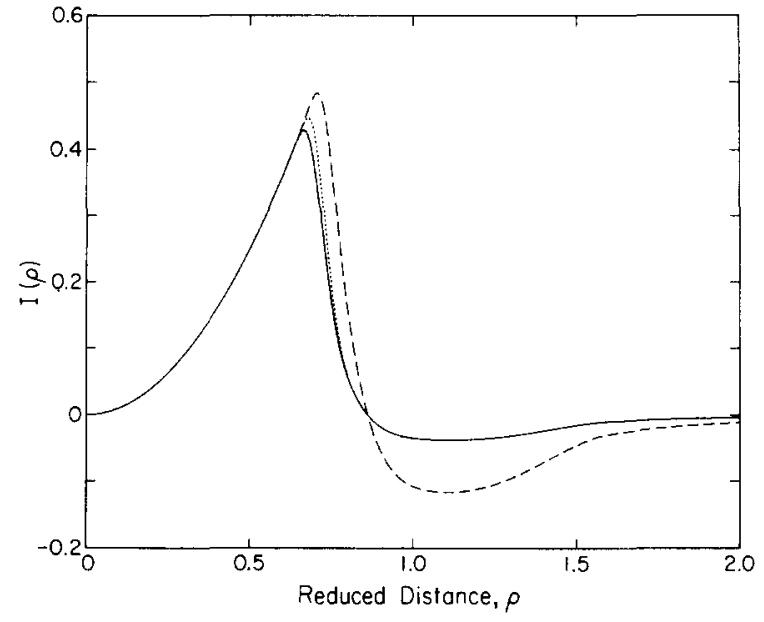

FIG. 7. Differential contribution to the classical second virial coefficient as a function of the reduced interatomic distance $\rho$. $I(\rho)$ is the integrand of the second virial coefficient expression [see Eq. (9) of the text]. Curves are calculated from the SPFD potential whose parameters are listed in Table II. Solid line: $T=600{ }^{\circ} \mathrm{K}$ and $\rho_{i}=0.80$; dashed line: $T=200^{\circ} \mathrm{K}$ and $\rho_{i}=0.80$; dotted line: $T=600^{\circ} \mathrm{K}$ and $\rho_{i}=0.81 ; \rho_{i}$ is the point below which a Born-Mayer repulsive wall is used as discussed in Sec. IV.

fit the strongly repulsive region of the interatomic potential to $B(T)$ data, having already fit the weakly repulsive and attractive region to their thermal DCS measurements. They accomplished this by using the ESMSV form ${ }^{55}$ to decouple these two regions at $\rho$ $\simeq 0.84(r=2.97 \AA)$. Dilute gas transport data are in principle even less sensitive to the (shallow) attractive well region of the $\mathrm{He}-\mathrm{Ar}$ potential than are $B(T)$ data when, as in the present case, $k T \gg \epsilon^{1 \mathrm{c}, 59}$; we will therefore use the repulsive wall part of the potential previously determined from these macroscopic properties, with no adjustments (see Sec. VI). The resulting potential, whose attractive and weakly repulsive regions had been obtained from the DCS fits, will be shown in Sec. VI to be consistent with all the available microscopic and macroscopic experimental data. We emphasize that the latter type of data, being insensitive to the attractive portion of the potential, must not be used in its characterization. For example, assuming a given mathematical form for the potential (such as a LJ12-6) allows a determination of $r_{m}$ and $\epsilon$ parameters by fitting to the macroscopic properties being considered. However, the validity of such a potential should not be considered to extend to the attractive region; these $r_{m}$ and $\epsilon$ therefore cannot be used as physically meaningful values for the position and depth of the well minimum. ${ }^{14,26}$ This is of course in strong contrast to DCS data which, at least for the present case, can be used to determine $r_{m}$ and $\epsilon$ values accurately. ${ }^{13}$

For any direct comparison of DCS scattering results between different laboratories to be useful, a large variety of involved apparatus characteristics (see Sec. II for examples) must be taken into consideration. In view of the difficulties inherent in such a procedure, and the fact that it is the potentials extracted from the data that are ultimately to be discussed, we use the approach of comparing the various laboratories' poten- 


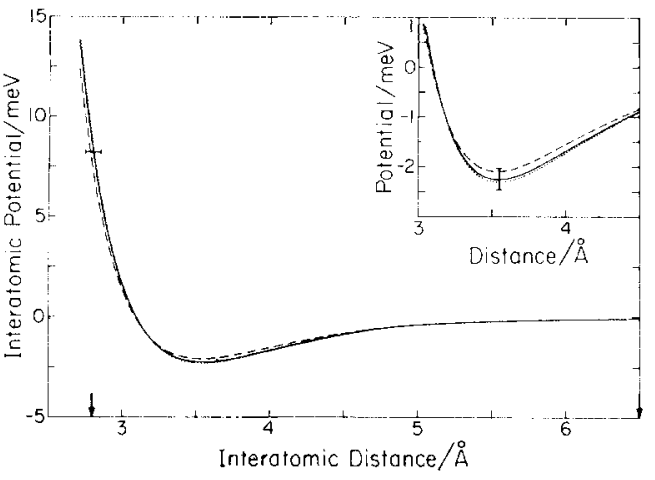

FIG. 8. Interatomic potentials of the MSV shape for He-Ar as a function of interatomic distance; inset as in Fig. 6. Solid curve: this work; dashed curve: Ref. 14; dotted curve: Ref. 15. The potential parameters are given in Table III. Error bars and arrows along the abscissa are placed as in Fig. 6 .

tials instead. ${ }^{14-16}$ This must be performed using the same potential form to fit all of the DCS experiments, since the simulation results of Paper I clearly show that in some instances the extracted potentials may depend strongly on the potential model used. ${ }^{13}$ Consequently, even though the MSV form ${ }^{52,55}$ previously used by others is not appropriate for obtaining accurate interatomic potentials from the present room-temperature scattering data ( $\mathrm{cf}$. the $\mathrm{M}^{2} \mathrm{SV}$ and SPFD potentials of Table II), it was used to reduce our data also, in order to compare respective scattering results.

Chen et al. 's ${ }^{14}$ experiments covered the laboratory angular range between $3^{\circ}$ and $45^{\circ}$ at a relative collision energy of $20.8 \mathrm{meV}$, while Smith $e t$ al. ' $^{15,16}$ results at $18.2 \mathrm{meV}$ were for scattering angles between $4^{\circ}$ and $30^{\circ}$. Although these collision energies are but a third of ours, the increased sensitivity of their DCS's to the long-range region of the potential is probably compensated for by our more extensive measurements of lowangle scattering; in any case, the long-range region of the potential is taken to be a theoretically estimated one and is not fit to the data. ${ }^{14-16}$ Conversely, the higher scattering angle but lower energy results are less sensitive to repulsive interactions than our higher energy but lower scattering angle measurements. ${ }^{13}$ We would therefore expect all three DCS's to be sensitive to the potential for interatomic distances between about 3.0 and $6.5 \AA$, as determined from the simulations of Paper I. The consistency of the three experiments may thus be judged by comparing, for this range of interatomic separations, the MSV potentials extracted from fits to the respective DCS's. From Fig. 8 it is evident that these potentials are indistinguishable within our precision limits, estimated in Paper I as $\pm 30 \%$ for the repulsive wall (corresponding to $\pm 0.04 \AA$ in the position of the wall), $\pm 10 \%$ for the attractive well, and $\pm 20 \%$ for the attractive tail. Because of the greater sensitivity of our room-temperature experiments to the weakly repulsive wall of the potential $(2.8 \AA \approx r \approx 3.1 \AA$ ), the MSV fit to our data tends to yield a slightly deeper well than it would if the wide-angle scattering data responsible for this sensitivity $\left(\theta>15^{\circ}\right)$ were neglected. However, the simulation calculation ${ }^{13}$ indicate that this
TABLE III. Fitted parameters for various MSV potentials. ${ }^{2}$

\begin{tabular}{lllcl}
\hline \hline$r_{m} / \AA$ & $\epsilon / \mathrm{meV}$ & $\chi^{2 ~ b, c, d}$ & $\Delta \alpha_{0.95} / \alpha{ }^{\mathrm{c}}(\%)$ & $\overline{R e f .}$ \\
\hline $3.54 \pm 0.01$ & $2.09 \pm 0.15$ & $4100(3)$ & 2.44 & 14 \\
3.55 & 2.30 & $3900(2)$ & 2.36 & 16 \\
3.55 & 2.25 & $3900(2)$ & 2.36 & $\begin{array}{l}\text { this } \\
\text { work }\end{array}$ \\
\hline \hline
\end{tabular}

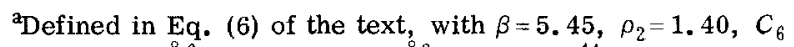
$=5.889 \mathrm{eV} \AA^{6} ; C_{8}=11.12 \mathrm{eV} \AA^{8} ; C_{10}=0.0 . .^{14}$

${ }^{\mathrm{b}} \mathrm{Scattering}$ intensity normalized to 500 an $\theta_{h}=0.0^{\circ}, \theta_{v}=4.9^{\circ}$ for the combined data fits; the number of parameters varied is given in parentheses.

${ }^{\text {c}}$ See Eqs. (1) and (3).

${ }^{\mathrm{d}}$ The fitted parameters were $\beta, r_{m}$, and $\epsilon$ for Ref. 14. For Ref. 15 and the present work they were $r_{m}$ and $\epsilon$ only, with $\beta$ fixed at the value obtained in Ref. 14 .

effect is $<10 \%$, so that correcting for it would not significantly affect the agreement among the curves of Fig. 8. Further documentation of the data's consistency from the three different laboratories is furnished in Fig. 9 where the DCS's calculated from the various MSV potentials are compared. In Table III we list the corresponding $r_{m}$ and $\epsilon$ parameters, and the goodnessof-fit statistical indices [Eqs. (1) and (3)], for the three calculated DCS's scaled to our combined in- and out-ofplane data. The quality of these three fits is substantially the same. Having thus established the consistency, and from it the reliability of the three different laboratories' scattering data, we may now apply the results of Paper I in selecting the most suitable $\mathrm{He}-\mathrm{Ar}$ potential. In Fig. 10 we display those $\mathrm{He}-\mathrm{Ar}$ potentials extracted from DCS fits and preferred by the three different laboratories: exp-6, ${ }^{16} \mathrm{HFD},{ }^{15} \mathrm{ESMSV},{ }^{14} \mathrm{M}^{2} \mathrm{SV}$, and SPFD. We now proceed to discuss them.

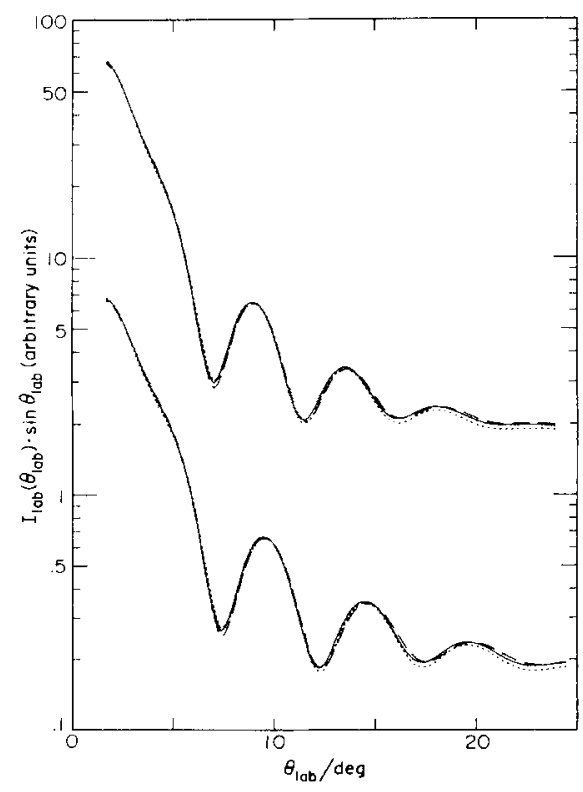

FIG. 9. Laboratory-averaged differential cross sections for in-plane and out-of-plane scattering calculated from the corresponding MSV potentials of Fig. 8. 


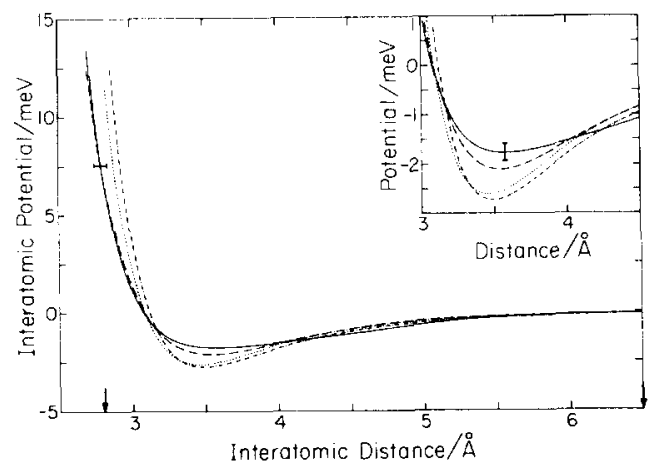

FIG. 10. Interatomic potentials proposed for He-Ar as a function of interatomic distance; inset as in Fig. 6. Solid curve: SPFD potential of this work; dashed curve: MSV potential from Ref. 14; dotted curve: HFD potential of Ref. 16; dash-dotted curve: exp-6 potential of Ref. 15. The $\mathrm{M}^{2} \mathrm{SV}$ potential is not shown here as it is very close to the SPFD one (see the solid curves of Fig. 6). Error bars and arrows along the abscissa are placed as in Fig. 6 .

The simulation calculations of Paper I showed that a given mathematical model must have sufficient flexibility to decouple different regions of the potential. ${ }^{13}$ This is required to enable an accurate representation of the interaction energy to be obtained by iteratively inverting the kinds of DCS data under discussion here. That is, each individual parameter should be related (in the ideal case) to a specific region of the potential, and conversely. ${ }^{12}$ The consequent unsuitability of the exp 6 potential [Eq. (5)] for fitting DCS data is substantiated by the results of Paper I. The single reduced form parameter $\alpha$ has the onus of determining the shape of the entire potential, including the long-range dispersion interaction for which it is known to be inappropriate at the distances sampled by the DCS experiments. ${ }^{18,54}$ On the other hand, the HFD formulation has a large number of parameters, each with a fairly clear physical interpretation. ${ }^{60,61}$ The resulting potential combines the convenience of being represented by a single analytical e:spression with the advantage of having ranges of interatomic distances in which the potential is sensitive to individual parameters. This pleasing combination is attained through use of exponential damping factors. We note that the HFD parameterization is a generalization of the Buckingham-Corner (BC) potential, ${ }^{10}$ and is somewhat akin to the BarkerPompe form, ${ }^{62}$ both of which were investigated in detail in Paper I. In fitting the potential to their DCS data, Smith et al.$^{15}$ varied only the $r_{m}$ and $\epsilon$ parameters of the HFD model; they relied on recent theoretically determined dispersion coefficients $C_{6}, C_{8}$, and $C_{10}{ }^{54}$ and on repulsion parameters fitted to $S C F-H F$ calculations. ${ }^{61}$ We shall hereafter refer to the HFD potential they obtained as "constrained" in the sense that the full flexibility of the HFD model was not exploited in the fits to their data. We note that the exponential repulsion parameters strongly influence the HFD potential ${ }^{15,60,61}$ at its attractive minimum and beyond, even though the HF calculations are unreliable at these large interatomic distances. ${ }^{61}$ For example, even at the minimum the contribution of this repulsion is $+2.4 \mathrm{meV}$, whereas that of the remaining attractive terms is $-5.0 \mathrm{meV}$.
It is apparent from these caveats that although the HFD form itself should indeed be highly flexible, ${ }^{15}$ the approximate nature of the data used in fixing the repulsion parameters of the constrained HFD potential restricts its flexibility to that of a two-parameter potential, and limits its ability to provide reliable $r_{m}$ and $\epsilon$ parameters. ${ }^{13}$ This is especially true because the repulsion parameters are responsible for determining not only the repulsive wall, but also the attractive well region of the potential, to which the DCS data are highly sensitive. As shown in Paper I and in Sec. IV of the present paper, a greater degree of flexibility is needed for an appropriate description of these regions of the potential. In particular, the simulation results of Paper I showed that the BC parameterization, having about the same flexibility as the unconstrained HFD form, ${ }^{15}$ does not yield an accurate long-range potential when fitted to the DCS data. By fixing this region of the potential with known values for the dispersion constants, ${ }^{54}$ as was done with the constrained HFD potential, the weakly repulsive wall and attractive minimum regions have no remaining flexibility. It is therefore not surprising that the $\mathrm{He}-\mathrm{Ar}$ constrained $\mathrm{HFD}^{15}$ potential is quite similar in shape to the rather inflexible ${ }^{13}$ exp-6 potential, ${ }^{16}$ as shown in Fig. 10. Nor is it surprising that both are quite different from the ESMSV potential, ${ }^{14}$ as well as from the present SPFD and $\mathrm{M}^{2} \mathrm{SV}$ potentials. Laboratory DCS curves corresponding to these proposed $\mathrm{He}-\mathrm{Ar}$ potentials are shown in Fig. 11. The SPFD potential form is fully able to mimic the HFD potential in direct numerical fits to the latter's shape ${ }^{13}$ [i. e., fitting only the $b_{0}$ and $b_{1}$ parameters of Eq. (8)], within the range of interatomic distances probed by the DCS experiments. Consequently, if the constrained HFD potential provides a description of the $\mathrm{He}-\mathrm{Ar}$ interaction within the accuracy obtainable from the scattering measurements, the present SPFD potential should

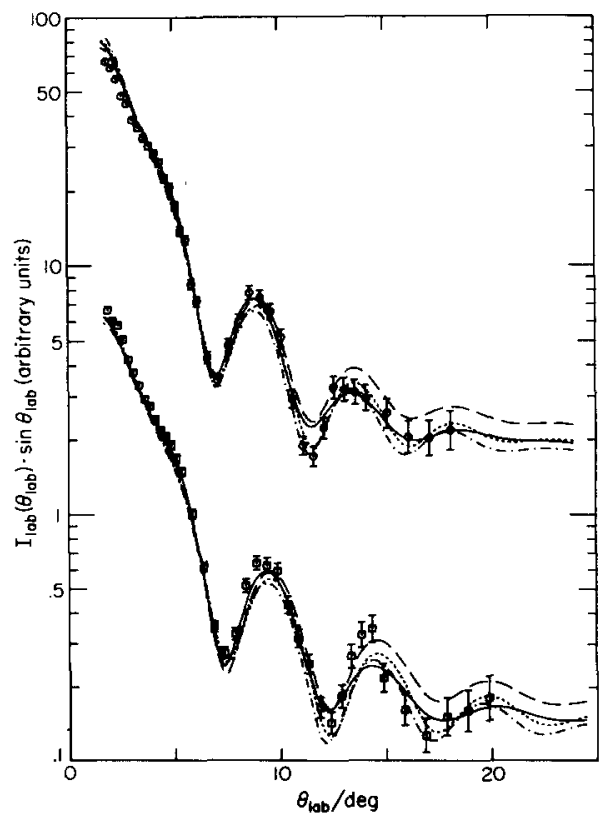

FIG. 11. Laboratory differential cross sections for in-plane and out-of-plane scattering. Points as in Fig. 4; curves are calculated from the corresponding potentials of Fig. 10 . 
be identical to it within that accuracy. ${ }^{13}$ However, as seen from Fig. 10, it is not. This is in spite of the equivalence of the scattering data used to obtain these respective potentials, as demonstrated earlier in this section (see also Figs. 8 and 9). From these considerations, we conclude that the constrained HFD potential does not provide an accurate representation of the He-Ar potential (see also Sec. VI. B).

In fitting the ESMSV potential to their scattering data, Chen et al. ${ }^{14}$ did not adjust its exponential or spline components; since these are used only for the strongly repulsive wall of the potential, their effect on the DCS fits are minimal. ${ }^{55}$ We can therefore use the simulation results of Paper I, as applied to the MSV potential form, ${ }^{52}$ to assess the suitability of the ESMSV form to provide a potential accurate in the van der Waals minimum region. The (ES)MSV potential is clearly superior to the exp 6 one, ${ }^{13}$ especially since it can be made to have the correct long-range behavior. With the availability of its Morse $\beta$ parameter to describe weakly repulsive interactions, the (ES)MSV potential may also be preferred over the constrained HFD one. However, as demonstrated in Paper I, the (ES)MSV parameterization is unable, for room-temperature experiments, to describe the attractive well region as adequately as some other potentials that have an additional parameter available to describe the weakly repulsive wall independently of the well region. This particular parametric independence does not appear to be required for the lower collision energy experiments. However, their increased sensitivity to the long-range region of the potential suggests that accurate values for the dispersion coefficients are particularly important. ${ }^{13}$ Thus, the use of a $C_{8}$ coefficient too weak by a factor of $2,{ }^{54}$ and the neglect of the $C_{10}$ contribution altogether, may

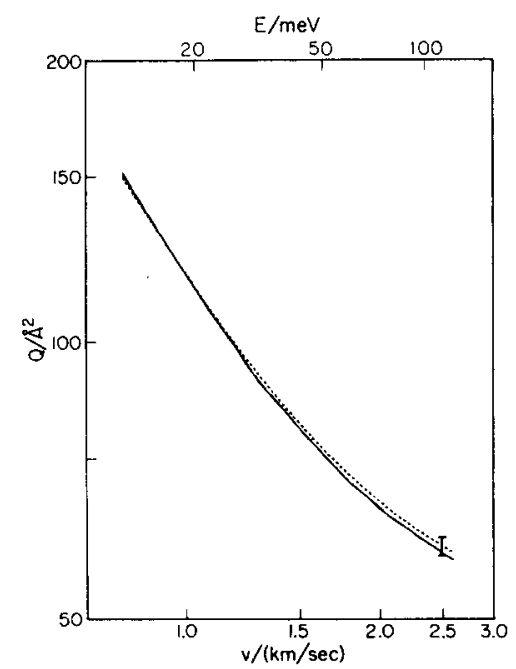

FIG. 12. He-Ar integral cross sections $Q$ as a function of the relative collision velocity $v$ (lower abscissa scale) and corresponding relative energy $E$ (upper abscissa scale). All scales are logarithmic. Solid curve: calculated from the SPFD potential of this work; dotted curve: calculated from the (ES) MSV potential of Ref. 17. The error bar at $v=2.5 \mathrm{~km} / \mathrm{sec}$ represents a constant calibration error of $\pm 2 \%$ in the experimental data of Ref. 17. induce a slight bias into the ESMSV potentials extracted from the low collision energy experiments. In addition, the dispersion series was used for distances that may be too close to the attractive minimum ${ }^{14}$ (see also Sec. VI. B).

We may use the above discussion to justify our preference for the shallower wells of our $\mathrm{M}^{2} \mathrm{SV}$ and SPFD potentials. However, because of the relatively small difference between those potentials and the ESMSV one, ${ }^{14}$ we cannot discriminate completely against the latter. Nevertheless, it must be stressed that the DCS data of the various laboratories, which are equivalent, should be reduced with more flexible potentials, or with potentials whose known parameters are more accurate than heretofore used. Otherwise, the analysis introduces a systematic bias into the potentials extracted from the data, ${ }^{13}$ a bias which may not be circumvented by claiming good fits of the calculated to the experimental DCS's. It is on this basis that we reject the exp- $6^{16}$ and the constrained HFD ${ }^{15}$ potentials as having attractive wells that are apparently too deep.

\section{APPROPRIATENESS OF THE $M^{2} S V$ AND SPFD POTENTIALS FOR THE DESCRIPTION OF OTHER PROPERTIES}

In this section, we compare the predictions of the SPFD or $\mathrm{M}^{2} \mathrm{SV}$ potentials, which are nearly equivalent (see Fig. 6), with experimental measurements of microscopic and macroscopic properties. We also refer to similar comparisons using the previously proposed scattering potentials. ${ }^{14-16}$

\section{A. Microscopic properties}

Integral cross sections $Q$, measured in arbitrary units as a function of the relative collision velocity $v$, provide data sensitive to the "area" of the attractive well. This area is usually expressed as a product of the $\epsilon$ and $r_{m}$ parameters, ${ }^{1 \mathrm{~b}}$ but is of course dependent upon the model chosen to describe the potential. The $\epsilon r_{m}$ product for an LJ12-6 model of the He-Ar interaction has been obtained from $Q(v)$ data as $7.7 \pm 0.8^{18}$ or $7.5 \pm 0.8^{63} \mathrm{meV} \AA$. To provide a first indication of the consistency of our DCS measurements with these results, both of which are sensitive to the attractive well region, we fitted the LJ12-6 model to our scattering data. We obtained an $\epsilon r_{m}$ product of $7.6 \mathrm{meV} \AA$, which is very close to the value obtained from the $Q(v)$ results. However, this value is no more reliable than is the rather crude LJ12-6 model, and is presented here only as a rough estimate of the compatibility of the (arbitrarily normalized) $Q(v)$ and DCS results. A more accurate value for the $\epsilon r_{m}$ product, obtained from the third-order SPFD and $\mathbf{M}^{2} \mathrm{SV}$ potentials (see Table III), is $6.4 \pm 0.7 \mathrm{meV} \AA$. A comparison of absolute $Q(v)$ values calculated directly from the respective SPFD and LJ12-6 potentials ${ }^{18}$ is given below.

More recent $Q(v)$ data have been obtained with absolute normalization, and have been used to determine the $r_{m}$ and $\epsilon$ parameters of an (ES)MSV potential. ${ }^{17}$ The potential obtained is identical to the (ES) MSV potential 
of Smith et al. ${ }^{15}$ and very close to our MSV potential; it is therefore significantly different from the more accurate third-order SPFD or $\mathrm{M}^{2} \mathrm{SV}$ potentials (see Figs. 8 and 10 Table IIJ). This equivalence of the several (ES)MSV potentials demonstrates the consistency of the (absolute) $Q(v)$ data with the DCS results. In Fig. 12, we compare $Q(v)$ curves calculated from the (ES)MSV potential as well as from the more accurate third-order SPFD and $\mathrm{M}^{2} \mathrm{SV}$ potentials [the $Q(v)$ curves for the $M^{2} S V$ and SPFD potentials are indistinguishable in the figure]. It can be seen that the curves are within the estimated systematic error of $\pm 2 \%$ in the absolute calibration of the $Q$. In addition, there seems to be an additional random experimental error not shown in the figure of about $\pm 2 \% .{ }^{17}$ The $Q(v)$ curve calculated from Helbing et al.'s LJ12-6 potential lies an average of $\sim 3 \%$ below that of the SPFD potential. ${ }^{18}$ These experiments are thus unable to discriminate between the potentials [i.e., the $Q(v)$ data are consistent with all of them], despite their sensitivity to the attractive well region $^{17}$ and the $30 \%$ greater well depth of the (ES)MSV potential. This is probably due to the $Q(v)$ data's insensitivity to the shape of the potential well; we emphasize again that the third-order SPFD or $\mathrm{M}^{2} \mathrm{SV}$ potentials are more accurate than is the (ES)MSV one, as demonstrated in Sec. V and Paper $I$.

By studying the vacuum ultraviolet absorption spectra of rare gases and their binary mixtures, the energy levels of many weakly bound van der Waals molecules have been obtained. ${ }^{19}$ Unfortunately, even a very recent study was unsuccessful in determining the dissociation energy for the $v=0$ vibrational level $D_{0}$ of either $\mathrm{He}-\mathrm{Kr}$ or $\mathrm{He}-\mathrm{Xe},{ }^{19}$ which probably supports only the $v=0$ level. ${ }^{64}$ To the best of our knowledge, no such studies are yet available for $\mathrm{He}-\mathrm{Ar}$. In anticipation of the future availability of such $D_{0}$ measurements, we compare below the calculated values for $D_{0}$ of the different $\mathrm{He}-\mathrm{Ar}$ potentials being considered. In Table IV we display these predictions for the SPFD and $M^{2} S V$ potentials, as well as those obtained from several previously proposed $\mathrm{He}-\mathrm{Ar}$ potentials. ${ }^{14-16}$ We also show the well depth $\epsilon$ and zero-point energy $G(0)$ for each of these potentials; none supports more than one vibrational level. Although the value of $\epsilon$ for the ESMSV potential is $\sim 17 \%$ greater than that for the SPFD or $\mathrm{M}^{2} \mathrm{SV}$ potentials, the respective $D_{0}$ values are all very close. Since $D_{0}=\epsilon-G(0)$, such fortuitous agreement is due to the larger curvature of the ESMSV minimum (listed as $\kappa$ in Table IV), which gives it a larger value of $G(0)$ than those of the SPFD and $M^{2} S V$ potentials (note that the latter two have similar values of $\kappa$ ). The $D_{0}$ values of the $\exp 6$ and HFD potentials are closer to those of the SPFD and $\mathrm{M}^{2} \mathrm{SV}$ potentials than are the respective well depths. Here, however, the compensatory effect of the greater curvature at the minima for the former two potentials than for the latter two is insufficient to produce a fortuitous agreement between these $D_{0}$ values.

Calculating $D_{0}$ for each of the third-order SPFD "experimental" potentials of Paper $I{ }^{13}$ we estimate the accuracy with which this quantity may be predicted for $\mathrm{He}-\mathrm{Ar}$ from DCS measurements to be about $\pm 5 \%$. The predicted dissociation energy determined from the potentials of this work is thus $D_{0}=(0.72 \pm 0.04) \mathrm{meV}$. The anticipated spectroscopic measurements of $D_{0}$ may eventually provide a very direct means of discriminating between some of the proposed potentials, at least in their respective attractive well regions. It should be noted, however, that such measurements cannot be used to obtain the well depth $\epsilon$ (equivalent to the dissociation energy $D_{e}$ ) of He-containing pairs, because the zeropoint energy may be estimated only if several vibrational states are observed. ${ }^{19,66,67}$ The implication is that for very weak attractive wells (those which support no more than two bound states), the DCS measurements contain more information about the shape of the well than do the spectroscopic measurements.

\section{B. Macroscopic properties}

In a preliminary comparison of experimental macroscopic dilute gas properties ${ }^{23-29}$ with those calculated from our $M^{2}$ SV or SPFD scattering potentials, we encountered a problem similar to that of Chen et al. ${ }^{14}$ They were unable to satisfactorily reproduce second virial coefficient $B(T)$ data with an MSV potential ${ }^{52}$ fitted solely to their $\mathrm{He}+\mathrm{Ar}$ scattering results, and chose to overcome this difficulty by using the ESMSV form $\mathrm{m}^{55}$ instead (see the beginning of Sec. V). This is quite reasonable insofar as the $B(T)$ data are more sensitive to the repulsive wall than are the scattering results; the former are sensitive primarily to the attractive portion of the potential only at low temperature (see Fig. 7 and Ref. 10). Viscosity and diffusion coefficients $\eta(T)$ and $D(T)$, respectively, are even less sensitive to the attractive portion of the potential ${ }^{10,59}$ in the relatively high temperature regime to which the experiments ${ }^{23-29}$ are inherently limited (by the condensability of the Ar); it is therefore to be anticipated that these properties may also not be described adequately by a potential fit solely to DCS measurements. The usefulness of the He-Ar $B(T), \eta(T)$, and $D(T)$ data therefore is not necessarily in distinguishing between potential curves or parameters characteristic of the attractive well and adjacent regions of the potential, but rather lies primarily in describing more strongly repulsive interactions. In this regard, these data provide a means of obtaining the potential for interatomic separations not probed by the thermal DCS measurements.

TABLE IV. Calculated spectroscopic quantities for $\mathrm{He}-\mathrm{Ar}$ from several porposed potentials. ${ }^{2}$

\begin{tabular}{llllll}
\hline \hline Potential & $\begin{array}{l}\epsilon / \\
\mathrm{meV}\end{array}$ & $\begin{array}{l}G(0) / \\
\mathrm{meV}\end{array}$ & $\begin{array}{l}D_{0} / \\
\mathrm{meV}\end{array}$ & $\begin{array}{l}\kappa / \\
\left(\mathrm{meV} / \AA^{2}\right)^{b}\end{array}$ & Ref. \\
\hline SPFD & 1.76 & 1.05 & 0.71 & 4.9 & this work \\
M $^{2} \mathrm{SV}$ & 1.80 & 1.08 & 0.72 & 5.2 & this work \\
ESMSV & 2.09 & 1.39 & 0.70 & 9.9 & 14 \\
HFD & 2.60 & 1.77 & 0.83 & 17.5 & 15 \\
exp-6 & 2.72 & 1.81 & 0.91 & 18.2 & 16 \\
\hline \hline
\end{tabular}

asee Ref. 65.

$b_{K}$ is defined as the curvature of the potential at the minimum of the attractive well. 
Dilute gas transport properties have been used to obtain the $\mathrm{He}-\mathrm{Ar}$ potential for repulsive interactions between 25 and $100 \mathrm{meV} .{ }^{2 B}$ The lower distance limit of $\sim 2.4 \AA$ to which these data are sensitive is governed by the availability of high-temperature diffusion data, while the upper distance limit is dictated by the failure of the fitting procedure for distances beyond $\sim 2.7 \AA$. The potential obtained was displayed graphically. ${ }^{26} \mathrm{We}$ have been able to represent it accurately and conveniently by the Born-Mayer form

$$
V_{\mathrm{BM}}(r)=C e^{-\alpha r} \text { for } 2.4<r<2.7 \AA \text {, }
$$

with the nonreduced values of $C=1.23 \times 10^{5} \mathrm{eV}$ and $\alpha$ $=5.69 \AA^{-1}$. The range of validity of this repulsive potential does not overlap that of the potentials obtained from fits to the scattering data (see Sec. V). Consequently, it should be possible to combine the potentials by "switching" between them. The "switch over" should, of course, occur for interatomic separations at which neither the DCS nor the $\eta(T)$ and $D(T)$ data are highly sensitive to the potential. Halfway between the upper limit for Eq. (10) of $\rho \simeq 0$. 75 and the lower reduced distance limit of sensitivity for DCS data $(\rho$ $\simeq 0.81)^{13}$ is therefore an appropriate central switch over distance $\rho_{0}$.

We choose a convenient switching function as

$$
h(\rho)=\frac{1}{1+e^{\tau\left(\rho-p_{0}\right)}} .
$$

This function rises from 0 to 1 as $p$ is decreased; the rapidity of the rise is governed by $\tau$. The switching function has a value $h(\rho)=0.5$ at $\rho=\rho_{0}$, so that we specify $\rho_{0}=0.78$. By requiring $h(\rho)=0.95$ at the upper limit for $\mathrm{Eq}$. (1) of $\rho=0.75$, thus dampening the influence of one region of the potential on properties depending on another, we specify $\tau=100$. The switching function is thus completely specified by the sensitivity of the DCS and gas transport properties to the potential, without adjustment of either of its parameters. It is used to combine the $V_{\mathrm{BM}}$ potential of Eq. (10) with the $\mathrm{M}^{2} \mathrm{SV}$ or SPFD potentials obtained by fits to the DCS measurements. Denoting the latter collectively as $V_{\mathrm{DCs}}$, the "modified" $\mathrm{M}^{2} \mathrm{SV}$ or SPFD potentials are given by

$$
V(r)=h\left(r / r_{m}\right) \times V_{\mathrm{BM}}(r)+\left[1-h\left(r / r_{m}\right)\right] \times V_{\mathrm{DCS}}(r) .
$$

The $r_{m}$ reduction parameter is 3.659 or $3.573 \AA$ for $V_{\text {DCS }}$ being the $\mathrm{M}^{2} \mathrm{SV}$ or SPFD potential, respectively (see Table II). In order to test the validity of the above procedure for combining scattering and macroscopic data, the modified $\mathbf{M}^{2} \mathrm{SV}$ and SPFD potentials are used to calculate values for DCS, transport, and equilibrium properties for comparison with experimental results.

The virial coefficients were calculated by numerically integrating Eq. (9); first and second quantum corrections $^{10}$ were calculated and applied to the classical $B(T)$, amounting to a total of $4.6 \%$ at $T=200^{\circ} \mathrm{K}$ and $1.0 \%$ at $T=600^{\circ} \mathrm{K}$. Transport coefficients were calculated in the first approximation ${ }^{10}$ by the method of Smith and Munn, ${ }^{68}$ but using quantum mechanical cross sections (see Sec. III). The experimental "interaction coefficients" $B_{12}, \eta_{12}$, and $D_{12}$, were calculated from mea- surements of the corresponding pure component and mixture virial and transport properties, with the exception of Brewer and Vaughn's coefficients. ${ }^{23}$ These were calculated using experimental literature values for the pure component $B(T)$.

The $\mathrm{M}^{2} \mathrm{SV}$ and SPFD potentials, whose parameters are listed in Table $I$, were modified using Eqs. (11) and (12), and then used for the bulk property calculations. Results are presented in Table $V$, showing the standard deviations of the various calculated properties from the corresponding experimental ones. Also shown in this table (see the last row) is the effect of modifying the $\mathrm{M}^{2} \mathrm{SV}$ and SPFD potentials on the standard deviations for the fits to our DCS data. These standard deviations correspond to values of the $\Delta \alpha_{0.95} / \alpha$ statistical index [Eq. (3)] of $2.16 \%$ and $2.15 \%$ for the modified $\mathrm{M}^{2} \mathrm{SV}$ and SPFD potentials, respectively. In comparing these $\Delta \alpha_{0.95} / \alpha$ to the values of $2.08 \%$ and $2.07 \% \mathrm{ob}-$ tained in the absence of the modifications (see Table II), their effect on the DCS is seen to be rather minimal. The standard deviations for our DCS fits may be scaled to those of other experiments by the signal intensities at our out-of-plane reference angle of $4.9^{\circ}$. Using the normalization appropriate for Smith et al.'s data, our standard deviation then becomes about 1.0 , which is of the same quality of their best fits to their data ${ }^{15}$ (Chen et al. do not report their fitting quality ${ }^{14}$ ).

Both the modified $\mathrm{M}^{2} \mathrm{SV}$ and SPFD potentials are seen to describe the experimental macroscopic data rather well (Table V). This is very encouraging, especially since the $B(T)$ data are reproduced, even though they were not used at all in choosing the modified potentials of Eq. (12). We also note from the table that the modified $\mathrm{M}^{2} \mathrm{SV}$ potential is slightly better able to describe the bulk property data than is the SPFD one.

The modified $\mathrm{M}^{2} \mathrm{SV}$ potential is shown in Fig. 13, which has a logarithmic energy scale above $1.0 \mathrm{meV}$. Also shown for comparison are the corresponding curves for the ESMSV potential, ${ }^{14}$ and for the HFD one. ${ }^{15}$ On the scale used, the modified SPFD potential is very close to the $\mathrm{M}^{2} \mathrm{SV}$ one and is not plotted. Let us first consider the three potentials in the repulsive region between 2.4 and $3.0 \AA$, where we can see that they are all quite similar. Both the modified $M^{2}$ SV and SPFD potentials describe $B(T)$ data as well as does the ESMSV one; this was the only bulk property considered by Chen et al. ${ }^{14}$ These two modified potentials also describe all the macroscopic data about as well as does the HFD potential ${ }^{15}$ (Table V). High energy integral collision cross section results are also shown in Fig. 13 for extremely repulsive interactions. ${ }^{69}$ Unfortunately, there is a gap between 1.8 and $2.4 \AA$ where the potential is poorly characterized by this method (or by any other); however, it is rather tempting to interpolate smoothly between the high-energy scattering and bulk property results. If such an interpolation is valid, the repulsive $\mathrm{He}$-Ar potential can now be regarded as reasonably well known for $r>1.4 \AA$.

The comparison among the three potentials in the attractive region is much less satisfactory. The dis- 
TABLE V. Standard deviations $\sigma$ for dilute gas properties.

\begin{tabular}{|c|c|c|c|c|c|c|}
\hline \multirow[b]{2}{*}{ Property } & \multirow[b]{2}{*}{$\begin{array}{l}\text { Temperature } \\
\text { range } /{ }^{\circ} \mathrm{K}\end{array}$} & \multirow[b]{2}{*}{ data range ${ }^{a-d}$} & \multicolumn{2}{|c|}{$\sigma$} & \multirow[b]{2}{*}{$\begin{array}{l}\text { Experimental } \\
\text { error }\end{array}$} & \multirow[b]{2}{*}{ Reference } \\
\hline & & & $\begin{array}{l}\text { modified } \\
\mathrm{M}^{2} \mathrm{SV}^{\mathrm{e}, \mathrm{P}}\end{array}$ & $\begin{array}{l}\text { modified } \\
\text { SPFD }\end{array}$ & & \\
\hline$B(T)^{b}$ & $123-323$ & $10.6-18.4(9)$ & 0.65 & 0.73 & 0.12 & 23 \\
\hline$B(T)$ & $223-323$ & $16.1-18.4(3)$ & 0.27 & 0.15 & 0.05 & 24 \\
\hline$B(T)$ & $303-773$ & $18.8-20.5(6)$ & 0.72 & 0.93 & 0.20 & 25 \\
\hline$\eta(T)^{\mathbf{c}}$ & $122-311$ & $88-160(8)$ & 2.5 & 4.1 & 2.5 & 26 \\
\hline$\eta(T)$ & $295-1553$ & $160-470(11)$ & 6.0 & 6.0 & 6.0 & 27 \\
\hline$D(T)^{\mathrm{d}}$ & $90-400$ & $0.09-1.2(4)$ & 0.034 & 0.031 & 0.003 & 28 \\
\hline$D(T)$ & $300-1400$ & $0.73-9.5(23)$ & 0.038 & 0.038 & 0.10 & 29 \\
\hline$B(T)^{\mathrm{b}}-$ total $^{\mathrm{g}}$ & $123-773$ & $10.6-20.5(18)$ & 0.60 & 0.71 & $\cdots$ & $\cdots$ \\
\hline$\eta(T)^{\mathrm{c}}$-total & $122-1553$ & $88-470(19)$ & 4.7 & 5.2 & $\cdots$ & $\cdots$ \\
\hline$D(T)^{\mathrm{d}}$-total & $90-1400$ & $0.09-9.5(27)$ & 0.036 & 0.037 & $\ldots$ & $\cdots$ \\
\hline $\mathrm{DCS}^{\mathrm{h}}$ & $\cdots$ & $9.8-4500(77)$ & 6.6 & 6.6 & $1.5-100$ & this work \\
\hline
\end{tabular}

${ }^{a}$ The number of data points that is compared with calculated values is given in parentheses; the data of Ref. 29 were "smoothed."

becond virial coefficients, units of $\mathrm{cm}^{3} /$ mole.

${ }^{c}$ Viscosity coefficients, units of $\mu \mathrm{P}$.

Diffusion coefficients at $1 \mathrm{~atm}$, units of $\mathrm{cm}^{3} / \mathrm{sec}$.

${ }^{8}$ Mathematical forms defined in Eqs. (7) and (8); potential parameters are given in Table II.

'The potentials are "switched over" to a Born-Mayer short-range repulsive wall as shown in Eqs. (11) and

(12). Parameters of Eq. (11) are $\tau=100$ and $\rho_{0}=0.78$; the nonreduced Born-Mayer parameters of Eq. (10) are $C=1.23 \times 10^{5} \mathrm{eV}$ and $\alpha=5.69 \AA^{-1}$.

g "total" applies to the combined data of the several references given for each particular property.

hDifferential cross sections in arbitrary units. Values of the $\Delta \alpha_{0.95} / \alpha$ statistical index of Eq. (3), corresponding to the absolute standard deviations quoted here, are $2.16 \%$ and $2.15 \%$ for the modified $M^{2} S V$ and SPFD potentials, respectively; cf. Table II. The DCS scattering intensities and experimental errors are normalized to 500 at the out-of-plane reference angle of $4.9^{\circ}$.

crepancies seen in Fig. 13, especially between the HFD and (modified) $\mathrm{M}^{2} \mathrm{SV}$ potentials, is beyond the $10 \%$ error bar established by independent computer simulation studies $^{13}$ for the sensitivity of the potentials in the attractive well region. These discrepancies are not caused by differences in the data, which have been shown to be equivalent (Sec. V). Nor can the discrepancies be resolved by appealing to macroscopic properties, which are described equivalently by our modified potentials and by Smith et al.'s HFD one (Table V and Ref. 16). Rather, the discrepancies are caused entirely by inadequacies in the potentials previously used to fit the scattering data. We have independently shown that the present $M^{2} S V$ and SPFD ones do have the flexibility required for an accurate potential to be obtained from iterative fits to the DCS data. ${ }^{13}$

As mentioned towards the middle of Sec. IV, we now discuss the choice of the reduced distance $\rho_{f}$ beyond which the theoretical multipole expansion is used $d^{54}$ for the SFPD potential [Eq. (8)]. For an early statistical model of interactions between closed-shell atoms and ions, it was found ${ }^{5 a}$ that the leading term of the multipole expansion mimics well the calculated potential for reduced distances beyond $\rho=1.1$ for $\mathrm{Ar}_{2}$ and $\rho=1.7$ for $\mathrm{He}_{2}$. Although the results of these calculations were not very reliable for the repulsive and for the attractive well regions of the potential, the relative contributions of the short-range, induction, and dispersion components to the potential at large interatomic separations is

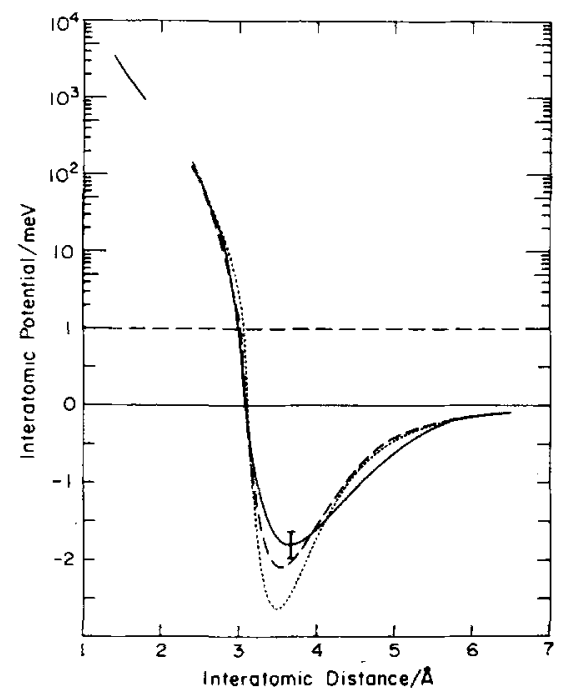

FIG. 13. He-Ar potentials, plotted semilogarithmically for energies above $1 \mathrm{meV}$, and linearly below $1 \mathrm{meV}$. Solid curve at the upper left is obtained from the high-energy integral collision cross section measurements of Ref. 69. Other curves are constructed from an amalgam of DCS and bulk property measurements. Solid curve: modified $\mathrm{M}^{2} \mathrm{SV}$ potential of this work (the modified SPFD potential is very similar and is not plotted); dashed curve: ESMSV potential of Ref. 14; dotted curve: HFD potential of Ref. 15. The horizontal error at $r=2.6 \AA$ is $\pm 0.01 \AA$ and is about the same as the horizontal displacements among the three potentials there ${ }^{26}$; at the attractive minimum, the error bar is $\pm 10 \%$ of the potential energy. ${ }^{13}$ 
TABLE VI. Fitted parameters for SPFD potentials with different values of $\rho_{f}{ }^{a}$

\begin{tabular}{lllllll}
\hline \hline$\rho_{f}{ }^{2, b}$ & $\gamma_{m} / \AA$ & $\epsilon / \mathrm{meV}$ & $b_{0}$ & $b_{1}$ & $\chi^{2 \mathrm{~b}, \mathrm{c}, \mathrm{d}}$ & $\Delta \alpha_{0.95} / \alpha^{\mathrm{d}(\%)}$ \\
\hline 1.4 & $3.72_{3}$ & 1.62 & 3.15 & 5.15 & $3100(4)$ & 2.12 \\
1.6 & $3.57_{3}$ & 1.76 & 17.8 & -4.69 & $2900(4)$ & 2.07 \\
1.8 & $3.59_{1}$ & 1.87 & 22.1 & -3.51 & $3000(4)$ & 2.11 \\
2.0 & $3.59_{0}$ & 2.05 & 24.7 & -3.19 & $3200(4)$ & 2.17 \\
1.64 & $3.58_{9}$ & 1.76 & 18.2 & -4.28 & $2900(5)$ & 2.07 \\
\hline
\end{tabular}

Reduced distance beyond which the potential assumes its theoretical ${ }^{54}$ asymptotic from [see Eq. (8)].

${ }^{b}$ For $\rho_{f}=1.4,1.6,1.8$, and 2.0, the fitted parameters were $r_{m}, \epsilon, b_{0}$, and $b_{1}$ of Eqs, (4) and

$(8)$; the $\rho_{f}=1.64$ value was obtained by optimizing it simultaneously with the other four parameters.

${ }^{\circ}$ Scattering intensity normalized to 500 at $\theta_{h}=0.0^{\circ}, \theta_{v}=4.9^{\circ}$; the number of parameters varied is given in parentheses for the fits to the "combined" data sets.

${ }^{\mathrm{d}}$ See Eqs. (1) and (3).

probably realistic. Our adoption of $\rho_{f}=1.6$ for switching to the first three terms of the multipole expansion is predicated upon these semiempirical results. ${ }^{5 a, 72}$ Lacking sufficiently detailed accurate theoretical calculations, we also seek to justify this choice empirically .

To this effect, we fit the SPFD potential $\left[r_{m}, \epsilon, b_{0}\right.$, and $b_{1}$ parameters of Eqs. (4) and (8)] to our DCS data for values of $\rho_{f}$ held fixed at 1.4, 1.8, and 2.0, in addition to the original value of 1.6. Now, the computer simulation results of Paper I showed that up to five statistically independent potential parameters may justifiably be fit to our DCS data, ${ }^{13}$ and that $\rho_{f}$ is one such parameter. We therefore use $\rho_{f}$ as the fifth parameter in place of the $c_{6}$ dispersion coefficient, ${ }^{53}$ which is held constant by using a fixed long-range potential (see Sec. IV), and fit the SPFD potential to the DCS data with the least-squares optimization of $\rho_{f}$ included. The parameters obtained for all these fits are shown in Table VI, and some of the resulting potentials are displayed in Fig. 14. Within the range of distances of the potential to which the DCS are sensitive, the effect of increasing $\rho_{f}$ is primarily to deepen the attractive well of the potential; the position of the repulsive wall is changed only slightly over the range of $\rho_{f}$ values considered. The shallow potential obtained from the $\rho_{f}=1.4 \mathrm{fit}$ is considered unrealistic because of its uneven shape in the interatomic distance region between 3.5 and $4.5 \AA$ (see the inset of Fig. 14); moreover, least-squares convergence was unusually difficult to attain. Both problems are caused by the small $b_{0}$ parameter value obtained, leading to inappropriately large ${ }^{73} b_{2}$ and $b_{3}$ coefficients. This empirical evidence that $\rho_{f}$ must be greater than 1.4 may indicate that the lower limit of interatomic distances for which the multipole expansion is valid is $\sim 5 \AA$ for $\mathrm{He}-\mathrm{Ar}$. For $\rho_{f}=2.0$, the increase in $\epsilon$ is seen to be beyond the error bar estimated to reflect the accuracy with which this part of the potential may be determined. ${ }^{13}$ A stronger indication that the most appropriate among the fixed $\rho_{f}$ values is $\rho_{f}=1.6$ is provided by the least-squares fitting of $\rho_{f}$ to the DCS data. As shown in Table VI, this results in a value of 1.64 ; the resulting potential is almost indistinguishable from the $\rho_{f}=1.6$ one and is therefore not plotted in Fig. 14.
In this section we have shown that the repulsive wall region of the potential, obtained exclusively from measurements of bulk properties, may be combined with the weakly repulsive and attractive regions of the potential, as determined exclusively by differential scattering experiments. Microscopic and macroscopic properties are simultaneously well described by this combined potential, which has thus been determined over a wider range of interatomic separations than would be possible by consideration of either microscopic or macroscopic properties alone. This is largely due to non-overlapping regions of sensitivity of such properties to the $\mathrm{He}-\mathrm{Ar}$ potential.

\section{SUMMARY AND CONCLUSIONS}

The computer simulation results of Paper I and the thermal DCS scattering measurements presented here have been used to construct two equivalent, accurate potentials, having different mathematical parameterizations, for the attractive well and adjacent regions of the

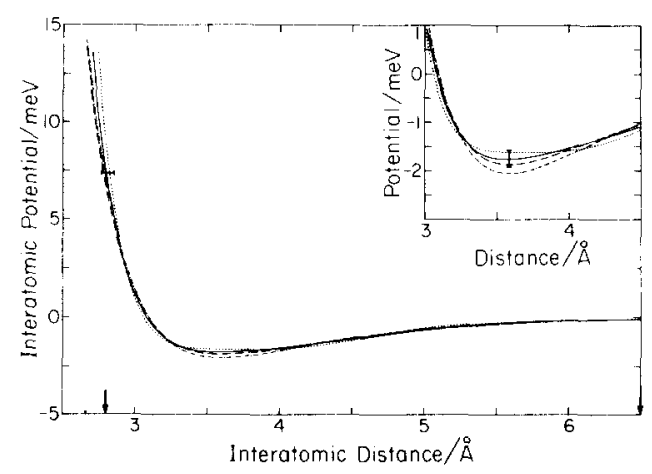

FIG. 14. Interatomic potentials of the SPFD shape for He-Ar as a function of interatomic distance; inset as in Fig. 6. Different curves are obtained fixing $f_{f}=1.6$ (solid); $\rho_{f}=1.8$ (dashed); $\rho_{f}=2.0$ (dash-dotted); $\rho_{f}=1.4$ (dotted), while fitting the four remaining SPFD parameters to DCS data. The corresponding curve with $p_{f}$ fitted along with the other SPFD parameters is almost indistinguishable from the $\rho_{f}=1.6$ curve and is not plotted. The potential parameters are given in Table VI. Error bars and arrows along the abscissa are placed as in Fig. 6. 
important heteronuclear rare gas diatom $\mathrm{He}-\mathrm{Ar}$. These potentials have been modified for strongly repulsive interactions, to which the DCS data are insensitive, by "switching over" to a potential determined exclusively from dilute gas transport properties. ${ }^{26}$ This modification has virtually no effect on the DCS scattering results, while a very good description of gas-phase bulk properties $^{23-29}$ is obtained without further variation of any parameters. Both potentials are most conveniently written in reduced form,

$$
f(\rho)=V(r) / \epsilon ; \rho=r / r_{m} .
$$

The first of these is the modified SPFD, ${ }^{21,22}$ written as

$$
\left.\begin{array}{l}
f(\rho)=h(\rho) A e^{-b \rho}+[1-h(\rho)]\left[b_{0} \lambda^{2}\left(1+\sum_{i=1}^{N} b_{i} \lambda^{i}\right)-1\right], \\
\text { with } h(\rho)=\frac{1}{\left.1+e^{\tau\left(\rho-\rho_{0}\right.}\right)} ; \lambda=1-1 / \rho \text { and } \rho<\rho_{f} \\
f(\rho)=-\sum_{i=1}^{3} c_{2 i+4} \rho^{-(2 i+4)} \text { for } \rho \geqslant \rho_{f} .
\end{array}\right\}
$$

We have used the results of Paper I to select $N=3 .^{13}$ The DCS data presented in Sec. IV and shown in Sec.V to be equivalent to those of two other laboratories, ${ }^{14,16}$ were used to determine the $r_{m}, \epsilon, b_{0}$, and $b_{1}$ parameters in the above equations. The value of the long-range

\begin{tabular}{|c|c|c|}
\hline Parameter & Value $^{b}$ & Method of determination \\
\hline$N$ & 3 & computer simulation ${ }^{\mathrm{c}}$ \\
\hline$r_{m}$ & $3.57_{3} \AA$ & DCS measurements $d$ \\
\hline$\epsilon$ & $1.76 \mathrm{meV}$ & DCS measurements ${ }^{d}$ \\
\hline$b_{0}$ & 17.75 & DCS measurements ${ }^{d}$ \\
\hline$b_{1}$ & -4.685 & DCS measurements ${ }^{d}$ \\
\hline$\rho_{f}$ & 1.60 & $\begin{array}{l}\text { semiempirically; DCS mea- } \\
\text { surements }\end{array}$ \\
\hline$c_{6}$ & 1.602 & theoretically ${ }^{p}$ \\
\hline$c_{8}{ }^{e}$ & 0.549 & theoretically \\
\hline$c_{10}{ }^{e}$ & 0.255 & theoretically \\
\hline$b_{2}$ & 15.13 & smoothness at $\rho=\rho_{f}$ \\
\hline$b_{3}$ & -19.23 & smoothness at $\rho=\rho_{f}$ \\
\hline$A^{\mathrm{B}}$ & $6.972 \times 10^{7}$ & viscosity; ${ }^{\text {h }}$ diffusion ${ }^{1}$ data \\
\hline$b^{\mathbf{g}}$ & 20.33 & viscosity $^{\text {h }}$ diffusion ${ }^{1}$ data \\
\hline$\rho_{0}$ & 0.78 & switching function [Eq. (11)] \\
\hline$\tau$ & 100 & switching function [Eq. (11)] \\
\hline
\end{tabular}
joining point $\rho_{f}$ was chosen on the basis of semiempirical calculations, ${ }^{5 a}, 72$ and was justified in Sec. VI by good-

TABLE VI. Modified SPFD potential parameters for He-Ar. ${ }^{\text {a }}$

${ }^{\text {a Modified SPFD form of Eq. (13), parameter symbols are as used }}$ there and in Eq. (4). $\sigma=3.08_{1} \AA$ for this potential.

bhose values quoted to four digits (except $r_{m}$ ) are not actually significant in the last place; we display then only to avoid roundoff errors which might otherwise result in apparent discontinuities of the potential at $\rho_{f}$.

'Reference 13.

dThis work.

${ }^{\theta}$ Nonreduced dispersion coeffcieints are ${ }^{53,54} C_{6}=5.867 \mathrm{eV} \AA^{6}$; $C_{8}=25.68 \mathrm{eV} \AA^{8} ; C_{10}=152.1 \mathrm{eV} \AA^{10}$.

${ }^{\prime}$ Reference 54.

${ }^{\mathrm{E}}$ Nonreduced Born-Mayer parameters are $\mathrm{C}=1.23 \times 10^{5} \mathrm{eV}$; $\alpha=5.69 \AA^{-1}$.

"References 26 and 27.

${ }^{1}$ References 28 and 29 . ness-of-fit criteria. For $\rho>\rho_{f}$, theoretical ${ }^{54}$ dispersion coefficients $c_{6}, c_{8}$, and $c_{10}$ were used; smoothness conditions at $\rho=\rho_{f}$ then determined $b_{2}$ and $b_{3}$. We obtained the Born-Mayer parameters $A$ and $b$ from a repulsive potential which resulted solely from gas transport properties. ${ }^{26}$ The "switching function" parameters $\rho_{0}$ and $\tau$ [see also Eq. (11)] were chosen to minimize the effect of one region of the potential on properties depending on the other. Integral cross section ${ }^{17,18,63}$ and virial coefficient $\mathrm{t}^{23-25}$ data were reproduced successfully by the modified SPFD potential, and were not considered further in its determination. Although our modified SPFD potential is characterized by a total of 15 parameters, only the five parameters $r_{m}, \epsilon, b_{0}, b_{1}$, and $\rho_{f}$ were obtained from the fits presented here. The final modified SPFD parameters are listed in Table VII.

The $r_{m}$ and $\epsilon$ parameters of course retain their familiar physical significance as the position and depth, respectively, of the attractive minimum, as do the dispersion coefficients ${ }^{53}$ and the Born-Mayer repulsive parameters $A$ and $b$. The $b_{0}$ parameter governs the curvature of the attractive minimum (i. e., $\left[d^{2} f / d \rho^{2}\right]_{\rho=1}$ $\left.=2 b_{0}\right)$, and $\rho_{f}$ may be regarded as the reduced distance beyond which the multipole expansion is appropriate. Unfortunately, the physical significance of the remaining parameters is more obscure.

The second of our accurate He-Ar potentials is the modified $\mathrm{M}^{2} \mathrm{SV},{ }^{20}$ written as:

$$
f(\rho)=h(\rho) A e^{-b \rho}+[1-h(\rho)] \frac{e^{\left(\beta^{\prime}-\rho \beta \omega\right)}}{\omega}-\left[e^{\left(\beta^{\prime}-\rho \beta \omega\right)}-2\right],
$$

where

$$
\begin{aligned}
& h(\rho)=\frac{1}{1+e^{\tau\left(\rho-\rho_{0}\right)}} ; \omega=\frac{\beta^{\prime}-\ln 2}{\beta-\ln 2} \text { and } \rho<1-\beta^{-1} \ln 2 \\
& f(\rho)=e^{\beta(1-\rho)}\left[e^{\beta(1-\rho)}-2\right] \text { for } 1-\beta^{-1} \ln 2 \leqslant \rho \leqslant \rho_{1} \\
& f(\rho)=\left(\rho_{2}-\rho\right)\left[s_{1}\left(\rho_{2}-\rho\right)^{2}+s_{3}\right]+\left(\rho-\rho_{1}\right)\left[s_{2}\left(\rho-\rho_{1}\right)^{2}+s_{4}\right] \\
& f(\rho)=-\sum_{i=1}^{3} c_{2 i+4} \rho^{-(2 i+4)} \text { for } \rho \geqslant \rho_{1}<\rho<\rho_{2} .
\end{aligned}
$$

The modified $\mathrm{M}^{2} \mathrm{SV}$ potential for $\mathrm{He}-\mathrm{Ar}$ is characterized by the 17 parameters listed in Table VIII. As for the modified SPFD potential [Eq. (13) and Table VII], only five of these parameters were adjusted to fit the DCS data: $r_{m}, \epsilon, \beta, \beta^{\prime}$, and $\rho_{2}$. Four of the remaining parameters are fixed by smoothness conditions $\left(s_{1}-s_{4}\right)$, and a fifth is otherwise completely determined $\left(\rho_{1}\right)$. The remaining long- and short-range parameters were obtained in the same fashion as were the corresponding modified SPFD parameters.

The large number of parameters required to adequately describe all the $\mathrm{He}-\mathrm{Ar}$ data discussed here indicates that it is highly unlikely that the principle of corresponding states ${ }^{10}$ can be used to predict anaccurate interatomic potential for this system from those of others. It is therefore also unlikely that combination rules can be found having a predictive accuracy comparable to that with which interaction potentials for sim- 
TABLE VIII. Modified $\mathrm{M}^{2} \mathrm{SV}$ potential parameters for He-Ar. ${ }^{a}$

\begin{tabular}{|c|c|c|}
\hline Parameter & Value $^{b}$ & Method of determination \\
\hline$r_{m}$ & $3.65_{9}$ & DCS measurements ${ }^{c}$ \\
\hline$\epsilon^{m}$ & $1.80^{\circ}$ & DCS measurements ${ }^{c}$ \\
\hline$\beta$ & 4.41 & DCS measurements ${ }^{c}$ \\
\hline$\beta^{\prime}$ & 8.54 & DCS measurements ${ }^{c}$ \\
\hline$\rho_{2}$ & 1.60 & $\begin{array}{l}\text { semiempirically; DCS measure- } \\
\text { ments }^{\mathrm{c}}\end{array}$ \\
\hline$c_{6}{ }^{d}$ & 1.358 & theoretically $^{e}$ \\
\hline$c_{8}{ }^{d}$ & 0.444 & theoretically ${ }^{e}$ \\
\hline$c_{10}{ }^{d}$ & 0.196 & theoretically \\
\hline$\rho_{1}$ & 1.157 & Morse $\beta$ parameter \\
\hline$s_{1}$ & -0.552 & smoothness at $\rho=\rho_{1}$ and $\rho_{2}$ \\
\hline$s_{2}$ & -2.573 & smoothness at $\rho=\rho_{1}$ and $\rho_{2}$ \\
\hline$s_{3}$ & -1.586 & smoothness at $\rho=\rho_{1}$ and $\rho_{2}$ \\
\hline$s_{4}$ & 0.294 & smoothness at $\rho=\rho_{1}$ and $\rho_{2}$ \\
\hline$A^{\mathrm{q}}$ & $6.834 \times 10^{7}$ & viscosity $^{\mathrm{g}}$; diffusion ${ }^{\mathrm{h}}$ data \\
\hline$b^{p}$ & 20.82 & viscosity $^{\mathrm{B}}$; diffusion ${ }^{\mathrm{h}}$ data \\
\hline$\rho_{0}$ & 0.78 & switching function [Eq. (11)] \\
\hline$\tau$ & 100 & switching function [Eq. (11)] \\
\hline
\end{tabular}

${ }^{\mathrm{a}}$ Modified $\mathrm{M}^{2} \mathrm{SV}$ form of Eq. (14), parameter symbols are as used there and in Eq. (4). $\sigma=3.08_{4} \AA$ for this potential.

${ }^{b}$ Those values quoted to four digits (except $r_{m}$ ) are not actually significant in the last place; we display them only to avoid round-off errors which might otherwise result in apparent discontinuities of the potential at $\rho=\rho_{1}, \rho_{2}$, or at $\sigma / r_{m}$.

cThis work.

${ }^{d}$ Nonreduced dispersion coefficients are ${ }^{53,54} C_{6}=5.867 \mathrm{eV}{ }^{6}$; $C_{8}=25.68 \mathrm{eV} \AA^{8} ; C_{10}=152.1 \mathrm{eV} \AA^{10}$.

${ }^{\circ}$ Reference 54 .

${ }^{1}$ Nonreduced Born-Mayer parameters are $C=1.23 \times 10^{5} \mathrm{eV}$; $\alpha=5.69 \AA^{-1}$.

${ }^{B}$ References 26 and 27.

"References 28 and 29 .

ple van der Waals systems may be experimentally determined.

Independent computer simulation studies ${ }^{13}$ indicated that the range of sensitivity of the present DCS data is 2.8 to $6.5 \AA$. Consequently, we consider this to be the minimum range of validity of the $M^{2} S V$ and SPFD potentials. However, in view of the supplementary information discussed here, the validity of the modified $\mathrm{M}^{2} \mathrm{SV}$ and SPFD potentials extends to all separations larger than $2.4 \AA$. If one furthermore interpolates these potentials over the 1.8 to $2.4 \AA$ gap for which no accurate information is available, the range of interatomic distances for which the $\mathrm{He}-\mathrm{Ar}$ potential can be assumed known is $r>1.4 \AA$. For such interatomic separations, these two potentials are identical within experimental error, and have been shown to provide an accurate representation of the He-Ar interaction. For distances $2.8 \approx r \approx 6.5 \AA$, the potentials obtained here are free of any siginificant biases arising either from the experimental data or from the parameterizations used to model the interaction.

\section{ACKNOWLEDGMENTS}

We wish to thank Messrs. W. W. Schuelke, A.W. Stark, and I. Moskovitz of the Instrument and Electronics Shops of the Division of Chemistry and Chemical Engineering at Caltech for their talented technical sup- port of the apparatus. We also wish to thank Ambassador College for generous use of its computing facilities.

\section{APPENDIX: c.m. TO LAB COORDINATE TRANSFORMATION}

We present here a brief description of the center of mass (c.m.) to laboratory (lab) coordinate transformation equations, for an arbitrary beam intersection angle and for arbitrary polar scattering angles. The quantities used in this derivation are defined in the velocity vector diagram of Fig. 15, which is restricted to elastic scattering, i.e.,

$$
|\mathbf{u}|=u=u_{1} \text {. }
$$

The $O z$ velocity-space lab coordinate axis is chosen to be coincident with the primary beam velocity vector, and the $x \geqslant 0 x z$ half-plane contains the secondary beam velocity vector. The $G Z$ velocity-space c.m. axis similarly coincides with the primary particle c. $m$. velocity vector with the $G X$ axis being in the $x \geqslant 0 x z$ half-plane. Orthogonal unit vectors in the lab frame are $\hat{\mathbf{i}}, \hat{\mathbf{j}}$, and $\hat{\mathbf{k}}$; in the c.m. frame they are $\hat{\mathbf{I}}, \hat{\mathbf{J}}$, and $\hat{\mathbf{K}}$. These unit vectors are related by:

$$
\left.\begin{array}{cc}
\hat{\mathbf{I}}=\hat{\mathbf{i}} \cos \alpha+\hat{\mathbf{k}} \sin \alpha & \text { (i) } \\
\hat{\mathbf{J}}=\hat{\mathbf{j}} & (\text { ii) } \\
\hat{\mathbf{K}}=-\hat{\mathbf{i}} \sin \alpha+\hat{\mathbf{k}} \cos \alpha & \text { (iii) }
\end{array}\right\},
$$

where $\alpha$ is easily expressed in terms of $v_{1}, v_{2}$, and $\gamma$. The relative collision velocity vector $u_{r}$ is given by

$$
\mathbf{u}_{r}=\mathbf{u}_{1}-\mathbf{u}_{2}=\mathbf{v}_{1}-\mathbf{v}_{2} \text {. }
$$

The scattered velocity vectors $\mathbf{v}$ and $\mathbf{u}$ are related by

$$
\mathbf{v}=\mathbf{v}_{\boldsymbol{g}}+\mathbf{u},
$$
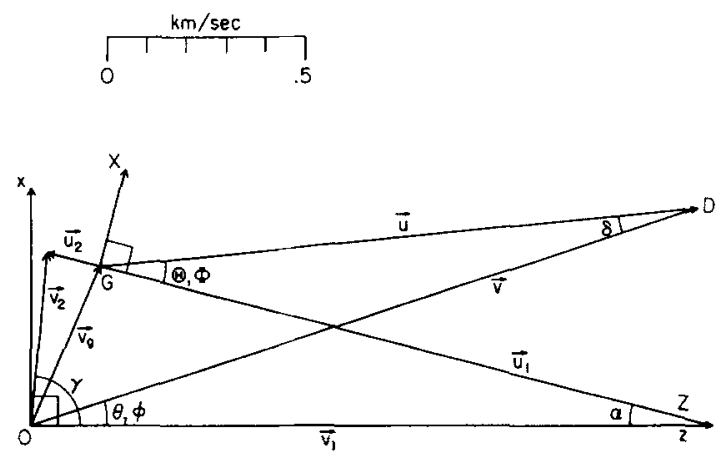

FIG. 15. Velocity vector diagram for He-Ar collisions at an arbitrary beam intersection angle $\gamma$. $O$ and $G$ are the origins (in velocity space) of the lab $\left(O_{x y z}\right)$ and c.m. (GXYZ) coordinate axes, respectively, as defined in the text. The detector position $D$ has spherical polar angles $\theta, \phi$ and $\theta, \Phi$ in these respective frames. In configuration space, the c.m. moves at a velocity $\mathrm{v}_{g}$ with respect to the lab origin; the $O z$ and $G Z$ coordinate axes intersect at an angle $\alpha$. Velocity vectors are denoted " $v$ " in the lab and " $u$ " in the c.m. frames; those with a subscript " 1 " refer to the primary beam, while a " 2 " refers to the secondary beam. The scattered velocity vectors $v$ and $u$ (no subscripts) intersect at an angle $\delta$. Symbols in the figure with overhead arrows are vector quantities, corresponding to boldface symbols in the text. 
where

$$
\mathbf{v}_{g}=\frac{1}{M}\left[\hat{\mathbf{k}}\left(m_{1} v_{1}+m_{2} v_{2} \cos \gamma\right)+\hat{\mathbf{i}} m_{2} v_{2} \sin \gamma\right]
$$

$M$ is the sum of the primary and secondary beam particle masses $m_{1}$ and $m_{2}$, respectively. The lab and c. m. scattered velocity vectors are given by

$$
\begin{aligned}
& \mathbf{v}=v(\hat{\mathbf{i}} \sin \theta \cos \phi+\hat{\mathbf{j}} \sin \theta \sin \phi+\hat{\mathbf{k}} \cos \theta) \quad(\mathrm{i}) \\
& \mathbf{u}=u(\hat{\mathbf{I}} \sin \theta \cos \Phi+\hat{\mathbf{J}} \sin \theta \sin \Phi+\hat{\mathbf{K}} \cos \theta) \quad \text { (ii) }
\end{aligned}
$$

From Eqs. (A2), (A4), (A5), and (A6) we obtain

$$
\begin{aligned}
& M v \sin \theta \cos \phi=m_{2}\left[v_{2} \sin \gamma+u_{r}(\sin \theta \cos \Phi \cos \alpha-\cos \theta \sin \alpha)\right] \\
& M v \sin \theta \sin \phi=m_{2} u_{r} \sin \Theta \sin \Phi \\
& M v \cos \theta=m_{1} v_{1}+m_{2}\left[v_{2} \cos \gamma\right. \\
& \left.\quad+u_{r}(\sin \theta \cos \Phi \sin \alpha+\cos \theta \cos \alpha)\right]
\end{aligned}
$$

It is now useful to express $\theta, \Phi$, and $v$ in terms of $\theta, \phi$, and $u_{\circ}$ Eqs。(A7i) and (A7ii) furnish

$$
\begin{array}{ll}
\Phi=0 & \text { for } \phi=0 \\
\Phi=\cos ^{-1}\left[\frac{r_{2} \sin \gamma(\cos \Theta-1)}{u_{r} \sin \Theta \cos \alpha}\right] & \text { for } \phi=\frac{\pi}{2} \\
\Phi=\cos ^{-1}\left[\frac{C_{\phi}\left(B_{\omega}^{2}+C_{\phi}^{2}-A_{\phi}^{2}\right)^{1 / 2}-A_{\phi} \dot{B}_{\phi}}{B_{\phi}^{2}+C_{\phi}^{2}}\right] & \text { for } \phi \neq 0, \neq \frac{\pi}{2}
\end{array}
$$

where

$$
\begin{aligned}
& A_{\phi}=v_{2} \sin \gamma \tan \phi(1-\cos \theta) \\
& B_{\phi}=u_{r} \sin \theta \tan \phi \cos \alpha \\
& C_{\phi}=u_{r} \sin \theta .
\end{aligned}
$$

The special cases $\phi=0$ and $\phi=\pi / 2$ correspond to in-plane and out-of-plane scattering, respectively, as defined in Sec. $\Pi$. We may similarly obtain $\theta$ (in the range 0 to $\pi$ ) from Eqs. (A7):

$$
\left.\begin{array}{ll}
\theta=\tan ^{-1}\left[\frac{m_{2}\left[v_{2} \sin \gamma(1-\cos \theta)+u_{r} \sin \Theta \cos \alpha\right]}{P_{k}}\right] & \text { for } \phi=0 \text { (i) } \\
\theta=\tan ^{-1}\left[\frac{m_{2} u_{r} \sin \Theta(1-\cos \phi)^{1 / 2}}{P_{k} \sin \phi}\right] & \text { for } \phi \neq 0(\mathrm{ii})
\end{array}\right\},
$$

where

$P_{k}=m_{1} v_{1}+m_{2}\left[v_{2}(\cos \gamma+\sin \Theta \sin \gamma)+u_{r} \cos \Theta \cos \alpha\right]$.

Finally, Eq. (A7iii) yields

$$
v=\frac{P_{k}}{M \cos \theta} \text {. }
$$

This quantity is useful in calculating the Jacobian of the $(\theta, \Phi)-(\theta, \phi)$ coordinate transformation. Requiring the conservation of flux upon performing this transformation, and relating differential surface elements in the two coordinate frames, the ratio of the lab to c.m. DCS gives ${ }^{74}$

$$
\frac{\sigma_{1 \mathrm{ab}}}{\sigma_{\mathrm{c.m} .}}=\frac{v^{2}}{u^{2}|\cos \delta|}
$$

The angle $\delta$ may be obtained from triangle OGD of Fig. 15 as

$$
\delta=\cos ^{-1}\left[\frac{u^{2}+v^{2}-v^{2}}{2 u v}\right] .
$$

The output signals of our electron-bombardment detector are proportional to number densities. To obtain quantities proportional instead to fluxes, and hence to DCS's, we must multiply those signals by the corresponding lab velocities. Therefore,

$$
\sigma_{1 \mathrm{ab}}=C \frac{I_{\mathrm{sc}} v}{I_{\operatorname{lnc}} v_{1}}
$$

where $I_{\mathrm{sc}}$ and $I_{\mathrm{inc}}$ are the scattered and incident signals, respectively. $C$ is independent of scattering direction, and includes the secondary beam number density and the detector acceptance angle. From Eqs. (A12) and (A14) we get

$$
I_{\mathrm{sc}}=\frac{\mathrm{C}}{I_{\mathrm{inc} \mid}} \sigma_{\mathrm{c} \cdot \mathrm{m} \cdot} \frac{v v_{1}}{u^{2}|\cos \delta|}
$$

where $C / I_{\text {inc }}$ is independent not only of the scattering direction, but also of $v$ and $v_{1}$.

After specifying the variables $v_{1}, v_{2}, \gamma, \phi$, and $\theta$, as well as the constants $m_{1}$ and $m_{2}$, the $(\Theta, \phi)-(\theta, \Phi)$ angular transformation is performed using Eqs. (A8) and (A9). The detected signal intensity $I_{s c}$ is then obtained (in arbitrary units) from Eqs. (A11) and (A15). 
${ }^{1}$ For recent reviews, see (a) U. Buck, Adv. Chem. Phys. 30, 313 (1975); (b) J. P. Toennies, in Physical Chemistry: an Advanced Treatise, Vol. 6A, edited by H. Eyring, D. Henderson, and W. Jost (Academic, New York, 1974), p. 227; (c) G. C. Maitland and E. B. Smith, Chem. Soc. Rev. 2, 181 (1973).

${ }^{2}$ I. Amdur and J. E. Jordan, Adv. Chem. Phys. 10, 29 (1966).

${ }^{3}$ A. A. Abrahamson, Phys, Rev. 130, 693 (1963).

${ }^{4}$ J. O. Hirschfelder and W. J. Meath, Adv. Chem. Phys. 12, 3 (1967).

5(a) Y. S. Kim and R. G. Gordon, J. Chem. Phys. 61, 1 (1974);

(b) J. S. Cohen and R. T Pack, ibid. 61, 2372 (1974); (c) P. D. Dacre, Chem. Phys, Lett. 50, 147 (1977).

${ }^{6}$ (a) A. L. J. Burgmans, J. M. Farrar, and Y. T. Lee, J. Chem. Phys. 64, 1345 (1976); (b) J. M. Parson, P. E. Siska, and Y. T. Lee, ibid. 56, 1511 (1972).

${ }^{7}$ D. E. Freeman, K. Yoshino, and Y. Tanaka, J. Chem. Phys. 61, 4880 (1974).

${ }^{8}$ See also Y. Tanaka, K. Yoshino, and D. E. Freeman, J. Chem. Phys. 59, 5160 (1973).

${ }^{9}$ See also G. L. Pollack, Rev. Mod. Phys. 36, 748 (1964)

${ }^{10} \mathrm{~J}$. O. Hirschfelder, C. F. Curtiss, and R. B. Bird, Molecular Theory of Gases and Liquids (Wiley, New York, 1954).

${ }^{11}$ For recently proposed combination rules, see for example (a) C. L. Kong and M. R. Chakrabarty, J. Phys. Chem. 77, 2668 (1973); (b) F. T. Smith, Phys. Rev. A 5, 1708 (1972); (c) H. L. Kramer and D. R. Herschbach, J. Chem. Phys. 53, 2792 (1970); (d) P. T. Sikora, J. Phys. B 3, 1475 (1970); see also Ref. 10 .

${ }^{12}$ Ch. Schlier, Ann. Rev. Phys, Chem. 20, 191 (1969).

${ }^{13} \mathrm{M}$. Keil and A. Kuppermann, J. Chem. Phys. 69, 3917 (1978).

${ }^{14}$ C. H. Chen, P. E. Siska, and Y. T. Lee, J. Chem. Phys. $59,601(1973)$.

${ }^{15}$ K. M. Smith, A. M. Rulis, G. Scoles, R. A. Aziz, and V. Nain, J. Chem. Phys, 67, 152 (1977).

${ }^{16}$ K. M. Smith, A. M. Rulis, G. Scoles, R. A. Aziz, and G. Duquette, J. Chem. Phys, 63, 2250 (1975).

${ }^{17}$ F. Pirani and F. Vecchiotcattivi, J. Chem. Phys. 66, 372 (1977).

${ }^{18}$ R. Helbing, W. Gaide, and H. Pauly, Z. Phys. 208, 215 (1968).

${ }^{19}$ D. E. Freeman, K. Yoshino, and Y. Tanaka, J. Chem. Phys. 67, 3462 (1977).

${ }^{20} \mathrm{Our} \mathrm{M}^{2} \mathrm{SV}$ potential is somewhat similar to the MMSV part of the ESMMSV potential form pooposed in Ref. 6(a). Their choice of joining the two Morse functions at $\rho=1$ was not used here since the first Morse function would represent both the weakly repulsive wall and a significant part of the attractive region, and therefore would not have the desired flexibility for fitting the DCS. Furthermore, we felt that the second Morse parameter (for $\rho \geq 1$ ) would then be used over too restricted a range of interatomic separations.

${ }^{21}$ G. Simons, R. G. Parr, and J. M. Finlan, J. Chem. Phys, 59, 3229 (1973).

${ }^{22}$ R. W. Bickes and R. B. Bernstein, Chem. Phys. Lett. 26, 457 (1974). Our SPF-Dunham potential is actually a further variant of their modification to the SPF potential. ${ }^{21}$ The major difference is the inclusion of dispersion terms of order higher than $C_{6} r^{-6}$, and in allowing these coefficients to be treated as parameters.

${ }^{23} \mathrm{~J}$. W. Brewer and G. W. Vaughn, J. Chem. Phys. 50, 2960 (1969).

${ }^{24}$ A. L. Blancett, K. R. Hall, and F. B. Canfield, Physica 47, 75 (1970).

${ }^{25}$ N. K. Kalfoglou and John G. Miller, J. Phys. Chem. 71, 1256 (1967).

${ }^{26}$ D. W. Gough, C. P. Matthews, and E. B. Smith, J. Chem. Soc. Faraday I 72, 645 (1976).

${ }^{27}$ G. C. Maitland and E. B. Smith, J. Chem. Soc. Faraday I 70, 1191 (1974).

${ }^{28}$ R. J. J. van Heijningen, J. P. Harpe, and J. J. M. Beenak- ker, Physica 38, 1 (1968).

${ }^{29}$ W. Hogervorst, Physica 51, 59 (1971).

${ }^{30}$ A. Kuppermann, R. J. Gordon, and M. J. Coggiola, Faraday Discuss. Chem. Soc. 55, 145 (1973); R. J。Gordon, M. J. Coggiola, and A. Kupperman, Chem. Phys. Lett. 20, 493 (1973).

${ }^{31}$ M. J. Coggiola, Ph. D. thesis, California Institute of Technology 1975

${ }^{32}$ J. B. Anderson and J. B. Fenn, Phys. Fluids 8, 780 (1965).

${ }^{33} \mathrm{H}$. U. Hostettler and R, B. Bernstein, Rev, Sci, Instrum.

31, 872 (1960).

${ }^{34}$ Galileo Electro-Optics Corporation, Sturbridge, Massachusetts.

${ }^{35}$ J. C. Johnson, A. T. Stair, and J. L. Pritchard, J. Appl. Phys. 37, 1551 (1966).

${ }^{36}$ Wallace and Tieman Company, Belleville, New Jersey.

${ }^{37}$ Bendix Corporation, South Montrose, Pennsylvania.

${ }^{38}$ Data Device Corporation, Bohemia, New York.

${ }^{39}$ N. F . Ramsey, Molecular Beams (Oxford University, Oxford, 1956), pp. 16-19; P. Kusch, J. Chem. Phys. 40, 1 (1964).

${ }^{40}$ G. O. Brink, Rev. Sci. Instrum, 37, 857 (1966).

${ }^{41}$ Extranuclear Laboratories Incorporated, P. O. Box 11512, Pittsburgh, Pennsylvania.

${ }^{42}$ Princeton Applied Research Corporation, P. O. Box 2565, Princeton, New Jersey.

${ }^{43}$ In addition to the primary and secondary beam angular divergence distribution function, a third one is needed to describe the overall apparatus angular resolution. Its FWHM is adjusted, as described in step $\mathbf{1 0}$ of Sec. III, and in Sec. IV. The calculated DCS is insensitive to the assumed shape of this apparatus angular resolution function; changing it from a cosine-squared to a hyperbolic secant affects the adjusted FWHM but not the calculated DCS. Since the overall apparatus angular resolution contains significant contributions from the angular distributions of the primary and secondary beams, we assume that this insensitivity to the exact form of the angular resolution function also applies to the primary and secondary beam angular distribution functions.

${ }^{44}$ Calculated by the method of R. T Pack, J. Chem. Phys. 60, 633 (1974). For $l \widetilde{>} 50$, JWKB phase shifts could be calculated more conveniently and uniformly by a slightly different Gauss-Mehler quadrature formula.

${ }^{45} \mathrm{~K}$. Smith, The Calculation of Atomic Collision Processes (Wiley, New York, 1971), p. 60. It was found that for the present single-channel case, the Numerov method was more accurate than that of R. G. Gordon, J. Chem. Phys. 51, 14 (1969); it was also about three times faster.

${ }^{46}$ R. B. Bernstein, J. Chem. Phys. 33, 795 (1960).

${ }^{47}$ M. Rosen and D. R. Yennie, J. Math. Phys. 5, 1505 (1964).

${ }^{48} \mathrm{H}$. M. Seip, in Selected Topics in Structure Chemistry, editeả by P. Andresen, O. Bastiansen and S. Furberg (Scandinavian University, Oslo, 1967), p. 25.

${ }^{49}$ N. R. Draper and H. Smith, Applied Regression Analysis (Wiley, New York, 1966), Chaps. 2 and 9.

${ }^{50}$ D. W. Marquardt, J. Soc. Indust. Appl. Math. 11, 431 (1963).

${ }^{51}$ R. B. Bernstein, Adv. Chem. Phys. 10, 75 (1966).

${ }^{52}$ J. M. Parson and Y. T. Lee, Entropie 42, 146 (1971). Modifications to their MSV potential concern the placement of the spline points; we have generally found that the spline region $\left(\rho_{1}<\rho<\rho_{2}\right)$ can best be freed of "wiggles" for a wide variety of $\beta, \rho_{2}$ and $c_{2 i+4}$ values if $\rho_{1}$ is chosen as in the text. ${ }^{53}$ The dispersion coefficients are written in reduced form as $c_{2 i+4}=\frac{C_{2 i+4}}{\epsilon r_{m}^{2 i+4}}(i=1,2,3)$.

${ }^{54}$ K. T. Tang, J. M. Norbeck, and P. R. Certain, J. Chem. Phys. 64, 3063 (1976).

${ }^{55}$ P. E. Siska, J. M. Parson, T. P. Schafer, and Y. T. Lee, 
J. Chem. Phys. 55, 5762 (1971). The ESMSV parametrization produces calculated DCS's which are almost identical to the corresponding MSV potentials.

${ }^{56}$ Other potential forms investigated in Paper I were the LJ12-6, LJn-6, Kihara $n-6$, Klein-Hanley, BuckinghamCorner, and double-LJ families, as well as the BarkerPompe expansion model; see Paper I for their explicit parametrizations.

${ }^{57}$ For additional functional forms that look interesting, see (a) A. J. Thakkar, J. Chem. Phys. 62, 1693 (1975); (b) G. C. Maitland and E. B. Smith, Chem. Phys. Lett. 22, 443 (1973); (c) V. H. Smith and A. J. Thakkar, ibid. 17, 274 (1972).

${ }^{58}$ We note that although neither of the potentials obtained separately were given any preference in this study, the outof-plane potential may actually be slightly preferred, primarily for two reasons. Firstly, angular spacings between the diffraction oscillations are greater for the out-of-plane DCS than for the in-plane one, allowing a better characterization of these oscillations. Secondly, the fits to the out-ofplane data are consistently of higher quality than are the inplane fits (see Fig. 4 and Table II). This difference may be related to small residual errors in the apparatus alignment (Sec. II). See also E. F. Greene, M. H. Lau, and J. Ross, J. Chem. Phys. 50, 3122 (1969).

${ }^{58}$ (a) J. H. Dymond and B. J. Alder, J. Chem. Phys. 51, 309 (1969); (b) Chem. Phys. Lett. 2, 54 (1968).

${ }^{60} \mathrm{~J}$. Hepburn, G. Scoles, and R. Penco, Chem. Phys. Lett. 36,451 (1975).
${ }^{61}$ R. Ahlrichs, R. Penco, and G. Scoles, Chem. Phys. 19, 119 (1977).

${ }^{62}$ J. A. Barker and A. Pompe, Aust. J. Chem. 21, 1683 (1968). ${ }^{63}$ R. Duren, R. Feltgen, W. Gaide, R. Helbing, and H. Pauly, Phys. Lett. 18, 282 (1965).

${ }^{64}$ M. V. Bobetic and J. A. Barker, J. Chem. Phys. 64, 2367 (1976).

${ }^{65}$ To integrate the $l=0$ radial wavefunction, we use the Numerov propagator instead of the Gordon method ${ }^{45}$ of Ref. 64; this introduces only a small change in the resulting $G(0)$ values $(<0.05 \mathrm{meV})$.

${ }^{66}$ (a) G. E. Ewing, Can. J. Phys. 54, 487 (1976); (b) G. E. Ewing, Accts. Chem. Res. 8, 185 (1975).

${ }^{67}$ Y. Tanaka and K. Yoshino, J. Chem. Phys. 53, 2012 (1970).

${ }^{68}$ F. J. Smith and R. J. Munn, J. Chem. Phys. 41, 3560 (1964).

${ }^{69}$ S. O. Colgate, J. E. Jordan, I. Amdur, and E. A. Mason, J. Chem. Phys. 51, 968 (1969).

${ }^{70}$ I. Amdur, E. A. Mason, and A. L. Harkness, J. Chem. Phys. 22, 1071 (1954).

${ }^{71}$ A. B. Kamnev and V. B. Leonas, Dokl. Akad. Nauk SSSR [Sov. Phys, -Dokladyl 10, 529 (1965).

${ }^{72}$ H. Kreek, Y. H. Pan, and W. J. Meath, Mol. Phys. 19, 513 (1970).

${ }^{73}$ J. M. Finlan and G. Simons, J. Mol. Spectrosc. 57, 1 (1975).

${ }^{74}$ (a) T. T. Warnock and R. B. Bernstein, J. Chem. Phys. 48, 1878 (1968); (b) R. K. B. Helbing, ibid. 48, 472 (1968); (c) F. A. Morse and R. B. Bernstein, ibid. 37, 2019 (1962). 